\title{
A new version of the convexification method for a 1-D coefficient inverse problem with experimental data* $^{*}$
}

\author{
Michael V. Klibanov ${ }^{\dagger \ddagger}$ Aleksandr E. Kolesov ${ }^{\dagger}$ Anders Sullivan, Lam Nguyen ${ }^{\ddagger}$
}

\begin{abstract}
A new version of the convexification method is developed analytically and tested numerically for a 1-D coefficient inverse problem in the frequency domain. Unlike the previous version, this one does not use the so-called "tail function", which is a complement of a certain truncated integral with respect to the wave number. Globally strictly convex cost functional is constructed with the Carleman Weight Function. Global convergence of the gradient projection method to the correct solution is proved. Numerical tests are conducted for both computationally simulated and experimental data.
\end{abstract}

Keywords. experimental data, coefficient inverse problem, convexification, Carleman Weight Function, numerical method, global convergence

AMS subject classification. 35R30, 78A46, 65C20

\section{Introduction}

In this paper, we develop a new version of the so-called convexification globally convergent numerical method for a 1-D coefficient inverse problem. Next, we demonstrate its performance for both computationally simulated and experimental data. The previous version of the convexification was applied by this research group to the same experimental data in [1. Rather than collecting the data in a laboratory, these data were collected in a realistic case of the cluttered environment in the field. The data collection was performed by the Forward Looking Radar of the US Army Research Laboratory [2]. The goal of this radar is to detect and identify flash explosive-like devices.

The radar community is currently relying only on the energy information of radar images, see, e.g. 3. Unlike this, in the current paper, so as in the previous one [1], we compute estimates of dielectric constants of targets. Our hope is that these estimates might help in the future to develop new classification

\footnotetext{
* Supported by US Army Research Laboratory and US Army Research Office grant W911NF-15-1-0233 and by the Office of Naval Research grant N00014-15-1-2330.

${ }^{\dagger}$ The corresponding author

${ }^{\ddagger}$ Department of Mathematics \& Statistics, University of North Carolina at Charlotte, Charlotte, NC 28223, USA (mklibanv@uncc.edu, akolesov@uncc.edu)

$\S$ U.S. Army Research Laboratory, Adelphi, MD 20783-1197 (lam.h.nguyen2.civ@mail.mil, anders.sullivan@us.army.mil)
} 
procedures, which would combine the currently used energy information with the estimates of dielectric constants. This combination, in turn might result in lower false alarm rates. Our targets are three dimensional ones of course. On the other hand, that radar can measure only one time dependent curve for each target. Thus, what can be done at most by any data inversion technique is to estimate a sort of an average of the dielectric constant for a target. We believe, however, that even these estimates might be useful for the goal of decreasing the false alarm rate. Thus, we model the wave propagation process by the 1-D Helmholtz equation.

Convexification is the method, which constructs globally strictly convex weighted Tikhonov-like functionals either for Coefficient Inverse Problems (CIPs) or for ill-posed Cauchy problems for quasilinear PDEs. See our works [1, 4, 5, 6. 7, 8, 9] for CIPs and [10, 11, 12, for quasilinear PDEs. The key element of such a functional is the presence of the Carleman Weight Function (CWF). This is the weight function in the Carleman estimate for the principal part of the corresponding differential operator.

Thus, convexification addresses the well known problem of multiple local minima and ravines of conventional Tikhonov-like functionals for CIPs, see, e.g. the paper 13 for a numerical example of multiple local minima. Convexification allows one to construct globally convergent numerical methods for CIPs. We call a numerical method for a CIP globally convergent if a theorem is proved, which claims that this method delivers at least one point in a sufficiently small neighborhood of the exact coefficient without any advanced knowledge of this neighborhood. Since conventional least squares Tikhonov functionals are non convex, then they usually have many local minima and ravines. This means that in order to obtain the correct solution, using such a functional, one needs to start the optimization process in a sufficiently small neighborhood of this solution, i.e. one should work with a locally convergent numerical method. However, such a small neighborhood is rarely known in applications.

The convexification is a globally convergent numerical method, see Remark 4.1 in section 4 . The idea of the convexification has roots in the method of Carleman estimates for CIPs. This method was originated in the work of Bukhgeim and Klibanov (1981) [14 as the tool of proofs of global uniqueness and stability theorems for CIPs with the data resulting from a single measurement event. The method of 14] became quite popular since then with many publications of many authors, see, e.g. a survey in [15], books [16, 17, 17] and references cited therein.

Another globally convergent numerical method for CIP with single measurement data is the so-called "tail functions" method, see, e.g. [16, 18. This method is currently completely verified on experimental data in a number of publications, see, e.g. [18 and reference cited therein.

The first publications on the convexification were [5, 6]. Some theoretical gaps, which prevented one from the active numerical studies, were fixed only recently in 10. As to the recent numerical results for the convexification for CIPs, we refer to [1, 8] for the 1-D case and to [9] for the 3-D case. Numerical results for the convexification for ill-posed Cauchy problems for quasilinear PDEs were published in [10, 12]. We also refer here to a recent interesting version of the convexification for a CIP for the equation $u_{t t}=\Delta u+a(x) u$ in which one of initial conditions at $\{t=0\}$ is not vanishing and the coefficient $a(x)$ is unknown, see 19. 
While, in the case of CIPs, all above references are for the data resulting from a single measurement event, in the paper 20 the data are generated by a point source running along an interval of a straight line. A new version of the convexification method is constructed in 20]. Detailed analytical and numerical studies of this version for the case of the inverse problem of electrical impedance tomography were recently conducted in 21 .

The new version of the convexification for the 1-D CIP of this paper is based on an adaptation of the idea of 20 for the case of our 1-D CIP. The governing PDE here is the 1-D Helmholtz equation, i.e. we work in the frequency domain. One of important new elements of [20] was the construction of a new orthonormal basis in $L_{2}(0,1)$ with some special properties properties. In [20] the functions of this basis were dependent on the position of the point source, and the same was in [21. However, unlike [20, 21], we do not move the source here.

Thus, a significantly new idea of the current publication is that we adapt that basis to the case when the wave number is running instead of the point source in 20. In addition, unlike 1, 9, the proof of the existence of the minimizer of our functional is different here from the one of [10. Along with Carleman estimates, we use here the apparatus of the convex analysis.

In addition to the new element mentioned in the preceding paragraph, we eliminate here the following two restrictive conditions of [1, 9]:

1. Most importantly, unlike [1, 9, we do not work here with a nonlinear integro differential equation in which the integration is carried out with respect to the wave number. This enables us to avoid the use of the so-called "tail function", which was used in [1, 9]. The tail function is the complement of a certain truncated integral. Since the tail function is unknown, a certain approximation for it was used in [1, 9].

2. Unlike [1, 9, we do not need to work here with large values of wave numbers.

CIPs have many applications in many different fields of science. Therefore, there is a large variety of publications devoted to numerical solutions of CIPs. We now refer to some of them as well as to the references cited therein [16, 22, $23,24,25,26,27,28,29,30$. As to another globally convergent numerical method for some CIPs, we refer to the technique of Kabanikhin and Shishlenin [31, 32, 33. This technique works with overdetermined data in the $n-\mathrm{D}$ case $(n=2,3)$, unlike the convexification.

In section 2 we state forward and inverse problems. In section 3 we construct our weighted Tikhonov-like functional. In section 4 we formulate our theorems. They are proved in section 5 . In section 6 we present our numerical results for both computationally simulated and experimental data.

\section{Statement of the inverse problem}

Below $k>0$ is the wave number. Also, for any $z \in \mathbb{C}$ we denote $\bar{z}$ its complex conjugation. 
Let $c_{0}>0$ be a positive number. Let $c(x), x \in \mathbb{R}$ be the function with the following properties:

$$
\begin{gathered}
c \in C^{2}(\mathbb{R}), \quad c(x) \geq c_{0}, \quad \forall x \in \mathbb{R}, \\
c(x)=1, \quad \forall x \notin(0,1),
\end{gathered}
$$

In our application $c(x)$ is the spatially distributed dielectric constant of the medium. Let $x_{0}<0$ be the position of the point source. The forward problem is:

$$
\begin{aligned}
u^{\prime \prime}+k^{2} c(x) u & =-\delta\left(x-x_{0}\right), \quad x \in \mathbb{R}, \\
\lim _{x \rightarrow \infty}\left(u^{\prime}+i k u\right) & =0, \lim _{x \rightarrow-\infty}\left(u^{\prime}-i k u\right)=0 .
\end{aligned}
$$

\subsection{Statement of the inverse problem}

Let $u_{0}(x, k)$ be the solution of the problem (3), (4) for the homogeneous case with $c(x) \equiv 1$,

$$
u_{0}(x, k)=\frac{e^{-i k\left|x-x_{0}\right|}}{2 i k} .
$$

In this paper we consider the following coefficient inverse problem:

Coefficient Inverse Problem (CIP). Let $[\underline{k}, \bar{k}] \subset(0, \infty)$ be an interval of wave numbers $k$. Reconstruct the function $c(x)$, assuming that the following function $g_{0}(k)$ is known

$$
g_{0}(k)=\frac{u(0, k)}{u_{0}(0, k)}, \quad k \in[\underline{k}, \bar{k}] .
$$

Uniqueness theorem for this CIP was proven in [34. Denote

$$
w(x, k)=\frac{u(x, k)}{u_{0}(x, k)} .
$$

By (6) and (7)

$$
w(0, k)=g_{0}(k), \quad k \in[\underline{k}, \bar{k}] .
$$

Besides, it was established in 34 that

$$
w^{\prime}(0, k)=g_{1}(k)=2 i k\left(g_{0}(k)-1\right), \quad k \in[\underline{k}, \bar{k}] .
$$

\subsection{Some properties of the solution of the forward prob- lem}

Lemma 2.1 is formulated in 34 as Lemma 4.1. However, since the proof was not provided in 34, we prove Lemma 2.1 here.

Lemma 2.1. The function $u(x, k) \neq 0$ for all $x>x_{0}$ and for all $k>0$. In particular, the function $g_{0}(k) \neq 0$ for all $k \in[\underline{k}, \bar{k}]$.

Proof. Since $x_{0}<0$ and by (2) $c(x)=1$ for $x \geq 1$, then (3) implies that $u^{\prime \prime}+k^{2} u=0$ for $x \geq 1$. Hence,

$$
u(x, k)=B_{1}(k) e^{i k x}+B_{2}(k) e^{-i k x}, \quad x \geq 1
$$


with some $k$-dependent complex numbers $B_{1}(k)$ and $B_{2}(k)$. Next, (4) implies that

$$
B_{1}(k)=0 .
$$

Assume that $u\left(x_{1}, k\right)=0$, where the point $x_{1} \in\left(x_{0}, 1\right)$. Multiply both sides of (3) by $\bar{u}(x, k)$ and integrate with respect to $x \in\left(x_{1}, 1\right)$. We obtain

$$
\left(u^{\prime} \bar{u}\right)(1, k)-\int_{x_{1}}^{1}\left|u^{\prime}\right|^{2} d x+k^{2} \int_{x_{1}}^{1} c(x)|u|^{2} d x=0 .
$$

By (10) and (11) $u^{\prime}(1, k)=-i k u(1, k)$. Substituting this in 12), we obtain

$$
-i k|u(1, k)|^{2}-\int_{x_{1}}^{1}\left|u^{\prime}\right|^{2} d x+k^{2} \int_{x_{1}}^{1} c(x)|u|^{2} d x=0 .
$$

Since the first term in the left hand side of $(13)$ is an imaginary number, whereas two other terms are real numbers, then (13) implies that $u(1, k)=0$. Hence, (10) and (11) imply that $u(x, k)=0$ for $x \geq 1$. Hence, the uniqueness result for the Cauchy problem for an ordinary ODE implies that

$$
u(x, k)=0 \text { for } x>x_{0} .
$$

Next, it follows from (2) and (3) that

$$
u(x, k)=B_{3}(k) e^{i k x}, \quad x<x_{0},
$$

where the complex number $B_{3}(k)$ depends only on $k$. It is well known (see, e.g. [35]) that the problem (3), (4) is equivalent with the 1-D Lippmann-Schwinger equation

$$
u(x, k)=\frac{e^{-i k\left|x-x_{0}\right|}}{2 i k}+\frac{k}{2 i} \int_{0}^{1} e^{-i k|x-\xi|}(c(\xi)-1) u(\xi, k) d \xi .
$$

Using (1) and (16), we obtain $u \in C(\mathbb{R}) \cap C^{3}\left(\mathbb{R} \backslash\left\{x_{0}\right\}\right)$. Hence, 14 implies that

$$
u\left(x_{0}, k\right)=0 .
$$

Hence, setting in 15 $x=x_{0}$, we obtain $B_{3}(k)=0$. The latter, 14, 15 and 17) imply that $u(x, k)=0$ for all $x \in \mathbb{R}$. This, however, is in the contradiction with the fact that the right hand side of equation (3) is not identical zero.

Lemma 2.1 helps us to define the function $\log w(x, k)$. The difficulty here is that the function $w(x, k)$ is a complex valued one. Hence, the ambiguity of $\log w(x, k)$ might be up to $2 n \pi i$, where $n$ is an integer. When defining $\log w(x, k)$, we follow [34. Consider the function $\phi(x)$,

$$
\phi(x)=-\frac{c^{\prime \prime}(x)}{c^{2}(x)}+\frac{7}{16} \frac{\left(c^{\prime}(x)\right)^{2}}{c^{3}(x)} .
$$

It was proven in theorem 2.2 of 34 that if

$$
\phi(x) \leq 0,
$$


then for $x>x_{0}$ the asymptotic behavior of the function $u(x, k)$ at $k \rightarrow \infty$ is

$$
u(x, k)=\frac{1}{2 i k c^{1 / 4}(x)} \exp \left[-i k \int_{x_{0}}^{x} \sqrt{c(\xi)} d \xi\right]\left(1+O\left(\frac{1}{k}\right)\right) .
$$

Assuming that this asymptotic behavior holds regardless on the inequality (18), we now remind the definition of 34 of the function $\log w(x, k)$. First, it follows from (5), (7) and (19) that we can define $\log w(x, k)$ for sufficiently large values of $k$ and for $x \in(0,1)$ as

$$
\begin{aligned}
& \log w(x, k)=-\frac{1}{4} \ln c(x)-i k\left(\int_{x_{0}}^{x} \sqrt{c(\xi)} d \xi-x+x_{0}\right) \\
&-\ln (2 k)-i \frac{\pi}{2}+\log (1+\widehat{w}(x, k))
\end{aligned}
$$

where

$$
\widehat{w}(x, k)=O\left(\frac{1}{k}\right), \quad k \rightarrow \infty .
$$

Hence, we set for sufficiently large $k$ :

$$
\log (1+\widehat{w}(x, k))=\sum_{n=1}^{\infty}(-1)^{n-1} \frac{(\widehat{w}(x, k))^{n}}{n},
$$

which eliminates the above mentioned ambiguity.

Let $K>0$ be such a number that 22 is valid for $k \geq K$. Note that $K$ might depend on $x, K=K(x)$. For $k \in(0, K)$ consider the function $\psi(x, k)$,

$$
\psi(x, k)=-\int_{k}^{\bar{k}} \frac{\partial_{k} w(x, \kappa)}{w(x, \kappa)} d \kappa+\log w(x, K),
$$

where $\log w(x, K)$ is defined via (20) and (22). By (7) and Lemma $2.1 w(x, \kappa) \neq$ $0, \forall x \in[0,1], \forall \kappa>0$. Differentiate both sides of (23) with respect to $k$. We obtain

$$
\partial_{k} w(x, k)-w(x, k) \partial_{k} \psi(x, k)=0 .
$$

Multiplying both sides of 24 by $\exp (-\psi(x, k))$, we obtain $\partial_{k}\left(e^{-\psi(x, k)} w(x, k)\right)=$ 0 . Hence, there exists a function $C=C(x)$ independent on $k$ such that

$$
w(x, k)=C(x) e^{\psi(x, k)} .
$$

Set in 25) $k=K$. By 23) $\psi(x, K)=\log w(x, K)$. Hence, we obtain from 25)

$$
C=C(x)=1, \quad x \in[0,1] .
$$

Hence, it follows from (25) and (26) that it is appropriate to define $\log w(x, k)$ as $\log w(x, k)=\psi(x, k)$. And ambiguity does occur this way.

\section{The Tikhonov-like Functional With the CWF}

In this section we construct the above mentioned weighted cost functional with the CWF in it. 


\subsection{A special orthonormal basis in $L_{2}(\underline{k}, \bar{k})$}

This basis was first constructed by Klibanov in [20]. In this reference, functions of that basis are dependent on the position of the point source, and this source runs along an interval of a straight line. We now briefly repeat that construction for the case when these functions depend on the wave number $k$. The latter is a new idea.

Let $($,$) denotes the scalar product in L_{2}(\underline{k}, \bar{k})$. We need to construct an orthonormal basis $\left\{\psi_{n}(k)\right\}_{n=0}^{\infty}$ of real valued function in the space $L_{2}(\underline{k}, \bar{k})$ such that the following two conditions are met:

1. $\psi_{n} \in C^{1}[\underline{k}, \bar{k}], \forall n=0,1, \ldots$

2. Let $a_{m n}=\left(\psi_{n}^{\prime}, \psi_{m}\right)$. Then the matrix $M_{N}=\left(a_{m n}\right)_{m, n=0}^{N-1}$ is invertible for any $N=1,2, \ldots$

Neither classical orthonormal polynomials nor the basis of trigonometric functions $\{\exp [i n \pi(k-\underline{k}) /(\bar{k}-\underline{k})]\}_{n=0}^{\infty}$ do not satisfy the second condition. This is because in any of these two cases the first column of the matrix $M_{N}$ would be equal to zero.

Consider the set of functions $\left\{k^{n} e^{k}\right\}_{n=0}^{\infty}$. This set is complete in $L_{2}(0,1)$. We orthonormalize it using the classical Gram-Schmidt orthonormalization procedure. We start from $n=0$, then take $n=1$, etc. Then we obtain the orthonormal basis $\left\{\varphi_{n}(k)\right\}_{n=0}^{\infty}$ in $L_{2}(0,1)$. Each function $\varphi_{n}(k)$ has the form

$$
\varphi_{n}(k)=p_{n}(k) e^{k},
$$

where $p_{n}(k)$ is the polynomial of the degree $n$. Next, we set

$$
\psi_{n}(k)=\frac{1}{\sqrt{\bar{k}-\underline{k}}} \varphi_{n}\left(\frac{k-\underline{k}}{\bar{k}-\underline{k}}\right) .
$$

Thus, the set $\left\{\psi_{n}(k)\right\}_{n=0}^{\infty}$ is an orthonormal basis in the space $L_{2}(\underline{k}, \bar{k})$. Lemma 3.1 ensures that the above property number 2 holds for functions $\psi_{n}(k)$.

Lemma 3.1 [20]. Denote $a_{m n}=\left(\psi_{n}^{\prime}, \psi_{m}\right)$. Then

$$
a_{m n}=\left\{\begin{array}{c}
(\bar{k}-\underline{k})^{-1} \text { if } n=m \\
0 \text { if } n<m
\end{array}\right.
$$

For an integer $N \geq 1$ consider the $N \times N$ matrix $M_{N}=\left(a_{m n}\right)_{(m, n)=(0,0)}^{(N-1, N-1)}$. Then (27) implies that $M_{N}$ is an upper diagonal matrix with $\operatorname{det}\left(M_{N}\right)=$ $(\bar{k}-\underline{k})^{-N} \neq 0$. Thus, the inverse matrix $M_{N}^{-1}$ exists.

\subsection{A system of coupled quasilinear ordinary differential equations}

Let $\log w(x, k)$ be the function constructed in section 2.2. Consider the function

$$
v(x, k)=\frac{\log w(x, k)}{k^{2}}, \quad x \in[0,1], k \in[\underline{k}, \underline{k}] .
$$


Substitution in equation (3) leads to

$$
v^{\prime \prime}+k^{2}\left(v^{\prime}\right)^{2}-2 i k v^{\prime}=1-c(x)=-\beta(x), \quad x \in[0,1], k \in[\underline{k}, \bar{k}] .
$$

Also, by (7)- 9

$$
v(0, k)=q_{0}(k), \quad v^{\prime}(0, k)=q_{1}(k),
$$

where

$$
q_{0}(k)=\frac{\log g_{0}(k)}{k^{2}}, \quad q_{1}(k)=\frac{g_{1}(k)}{g_{0}(k) k^{2}} .
$$

In addition, (10) and 11 imply that

$$
v^{\prime}(1, k)=0 .
$$

Differentiate both sides of equation $(29)$ with respect to $k$ and use the fact that $\partial_{k}(1-c(x))=0$. We obtain

$$
v_{k}^{\prime \prime}+2 k^{2} v_{k}^{\prime} v^{\prime}+2 k\left(v^{\prime}\right)^{2}-2 i k v_{k}^{\prime}-2 i v^{\prime}=0 .
$$

We now assume that the function $v(x, k)$ can be represented via a truncated Fourier series with respect to the orthonormal basis $\left\{\psi_{n}(k)\right\}_{n=0}^{\infty}$. In fact, this is our main approximation. Thus, for an integer $N \geq 1$, we assume that

$$
v(x, k)=\sum_{n=0}^{N-1} y_{n}(x) \psi_{n}(k)
$$

for $x \in[0,1], k \in[\underline{k}, \bar{k}]$. Here coefficients $y_{n}(x)$ of the expansion 34 are unknown and should be determined as the main part of the solution of our CIP. In particular, 29 and $(34)$ imply that

$$
\begin{gathered}
c(x)=1-\sum_{n=0}^{N-1} y_{n}^{\prime \prime}(x) \psi_{n}(k)-k^{2}\left(\sum_{n=0}^{N-1} y_{n}^{\prime}(x) \psi_{n}(k)\right)^{2} \\
+2 i k \sum_{n=0}^{N-1} y_{n}^{\prime}(x) \psi_{n}(k)
\end{gathered}
$$

Substituting (34) in (33), we obtain

$$
\begin{gathered}
\sum_{n=0}^{N-1} y_{n}^{\prime \prime}(x) \psi_{n}^{\prime}(k)+2 k^{2} \sum_{n, m=0}^{N-1} y_{n}^{\prime}(x) y_{m}^{\prime}(x) \psi_{n}^{\prime}(k) \psi_{m}^{\prime}(k) \\
+2 k\left[\sum_{n=0}^{N-1} y_{n}^{\prime}(x) \psi_{n}(k)\right]^{2}-2 i k \sum_{n=0}^{N-1} y_{n}^{\prime}(x) \psi_{n}^{\prime}(k) \\
-2 i \sum_{n=0}^{N-1} y_{n}^{\prime}(x) \psi_{n}(k)=0 .
\end{gathered}
$$

Introduce the $N-\mathrm{D}$ vector function $y(x)$,

$$
y(x)=\left(y_{0}, \ldots, y_{N-1}\right)^{T}(x) .
$$


Let $s \in[0, N-1]$ be an integer. Multiply both sides of $(36)$ by $\psi_{s}(k)$ and integrate with respect to $k \in(\underline{k}, \bar{k})$. We obtain

$$
M_{N} y^{\prime \prime}+\widetilde{F}\left(y^{\prime}\right)=0, \quad x \in[0,1] .
$$

Here the vector function $\widetilde{F}(\cdot)$ is quadratic with respect to the functions $y_{n}^{\prime}(x)$. Since by Lemma 3.1 the matrix $M_{N}$ is invertible, we multiply both sides of (38) by $M_{N}^{-1}$ and obtain

$$
y^{\prime \prime}+F\left(y^{\prime}\right)=0, \quad x \in[0,1],
$$

where the vector function $F\left(y^{\prime}\right)=M_{N}^{-1} \widetilde{F}\left(y^{\prime}\right)$. Therefore, the vector function $F\left(y^{\prime}\right)$ is quadratic with respect to the functions $y_{n}^{\prime}(x)$. In addition, (30) and (32) imply that $y(x)$ satisfies the following boundary conditions:

$$
y(0)=f_{0}, \quad y^{\prime}(0)=f_{1}, \quad y^{\prime}(1)=0 .
$$

Note that due to the boundary condition $y^{\prime}(1)=0,39,40$ is not the regular Cauchy problem for the system (39) of coupled quasilinear Ordinary Differential Equations. Thus, our effort below is focused on the numerical solution of the problem (39), (40). Indeed, if we would solve it, then, using (35) and (37), we would find the target coefficient $c(x)$. We solve this problem via minimizing our weighted Tikhonov-like functional with the CWF. First, we formulate the Carleman estimate for the operator $d^{2} / d x^{2}$.

Lemma 3.2 (Carleman estimate) [1]. For any complex valued function $u \in$ $H^{2}(0,1)$ with $u(0)=u^{\prime}(0)=0$ and for any parameter $\lambda \geq 1$ the following Carleman estimate holds

$$
\begin{gathered}
\int_{0}^{1}\left|u^{\prime \prime}\right|^{2} e^{-2 \lambda x} d x \geq C \int_{0}^{1}\left|u^{\prime \prime}\right|^{2} e^{-2 \lambda x} d x \\
+C\left[\lambda \int_{0}^{1}\left|u^{\prime}\right|^{2} e^{-2 \lambda x} d x+\lambda^{3} \int_{0}^{1}|u|^{2} e^{-2 \lambda x} d x\right],
\end{gathered}
$$

where the constant $C>0$ is independent on $u$ and $\lambda$.

Remark 3.1. Using Lemma 3.1 as well as arguments, which are completely similar with those of the proof of Theorem 3.1 of [20], one can prove that there exists at most one solution $V \in H^{2}(0,1)$ of the problem (39), (40).

\subsection{Weighted Tikhonov-like functional}

Introduce the cut-off function $\chi(x)$,

$$
\chi \in C^{2}[0,1], \quad \chi(x)=\left\{\begin{array}{c}
1, x \in[0,1 / 2], \\
0, x \in[3 / 4,1], \\
\in(0,1), x \in(1 / 2,3 / 4) .
\end{array}\right.
$$

Consider the $N-\mathrm{D}$ vector function $f(x)$ defined as

$$
f(x)=\left[f_{0}+x f_{1}\right] \chi(x) .
$$

Then

$$
f \in C^{2}[0,1], \quad f(0)=f_{0}, \quad f^{\prime}(0)=f_{1}, \quad f^{\prime}(1)=0
$$


Hence

$$
(y-f)(0)=0, \quad(y-f)^{\prime}(0)=0, \quad(y-f)^{\prime}(1)=0 .
$$

Let $R>0$ be an arbitrary number. Consider the sets $B\left(R, f_{0}, f_{1}\right)$ and $B_{0}(R)$ of $N-\mathrm{D}$ vector functions $W(x)$ and $p(x)$ defined as:

$$
\begin{gathered}
B\left(R, f_{0}, f_{1}\right)=\left\{\begin{array}{c}
W \in H^{2}(0,1): \\
W(0)=f_{0}, W^{\prime}(0)=f_{1}, W^{\prime}(1)=0, \\
\|W-f\|_{H^{2}(0,1)}<R
\end{array}\right\}, \\
B_{0}(R)=\left\{p \in H^{2}(0,1): p(0)=p^{\prime}(0)=p^{\prime}(1)=0,\|p\|_{H^{2}(0,1)}<R\right\} .
\end{gathered}
$$

Hence, all vector functions belonging to the set $B\left(R, f_{0}, f_{1}\right)$ satisfy boundary conditions 40$)$. Note that both sets $B\left(R, f_{0}, f_{1}\right)$ and $B_{0}(R)$ are convex. The proof of Proposition 3.1 follows immediately from $(44)$ and $(45)$.

Proposition 3.1. Let $f(x)$ be the vector function defined in (42). Then for any vector function $W \in B\left(R, f_{0}, f_{1}\right)$ the vector function $(W-f) \in B_{0}(R)$. And vice versa: for any vector function $p \in B_{0}(R)$ the vector function $(p+f) \in$ $B\left(R, f_{0}, f_{1}\right)$.

In accordance with the Tikhonov concept for ill-posed problems 36, we assume that there exists the exact solution $y^{*}$ of the problem (39), 40) with the exact (i.e. noiseless) data

$$
y^{*}(0)=f_{0}^{*}, \quad y^{* \prime}(0)=f_{1}^{*}
$$

in (40) and $y^{*} \in B\left(R, f_{0}^{*}, f_{1}^{*}\right)$. Let a sufficiently small number $\delta \in(0,1)$ represents the level of the noise in the data, i.e.

$$
\left|f_{0}-f_{0}^{*}\right|<\delta, \quad\left|f_{1}-f_{1}^{*}\right|<\delta .
$$

Similarly with 42 introduce the function $f^{*}(x)$,

$$
f^{*}(x)=\left[f_{0}^{*}+x f_{1}^{*}\right] \chi(x) .
$$

It follows from 41, 42 , 47) and (48) that

$$
\left\|f-f^{*}\right\|_{C^{2}[0,1]} \leq B \delta .
$$

Here and below $B=B(\chi)>0$ denotes different positive numbers depending only on the function $\chi(x)$. Since $\delta$ is sufficiently small, we indicate below dependencies of some constants on $f^{*}$ rather than on $f$.

Let $\alpha \in(0,1)$ be the regularization parameter. Our Tikhonov-like weighted functional is

$$
J_{\lambda, \alpha}(y)=e^{2 \lambda} \int_{0}^{1}\left|y^{\prime \prime}+F\left(y^{\prime}\right)\right|^{2} e^{-2 \lambda x} d x+\alpha\|y\|_{H^{2}(0,1)}^{2} .
$$

Here the multiplier $e^{2 \lambda}$ is introduced to balance two terms in the right hand side of (50). We consider the following minimization problem:

Minimization Problem. Minimize the functional $J_{\lambda, \alpha}(y)$ on the set $y \in$ $\overline{B\left(R, f_{0}, f_{1}\right)}$

Remark 3.1. In principle, estimate (51) of Theorem 4.1 tells one that it is not necessary to incorporate the regularization term $\alpha\|y\|_{H^{2}(0,1)}^{2}$ in the functional $J_{\lambda, \alpha}(y)$. Nevertheless we have observed in our computations that the presence of this term improves numerical results. This is why we introduce it here. We cannot yet explain the reason of this improvement. 


\section{Theorems}

In this section we formulate theorems about the functional $J_{\lambda, \alpha}(y)$. We prove them in section 5. Theorem 4.1 is the central result of this paper, also see Remark 3.1 about $\alpha$. Below $C_{1}=C_{1}(R, F, N)>0$ and $C_{2}=C_{2}\left(R, F, N, \chi, f^{*}\right)>$ 0 denote different numbers depending only on listed parameters.

Theorem 4.1. The functional $J_{\lambda, \alpha}(y)$ has the Frechét derivative $J_{\lambda, \alpha}^{\prime}(y)$ at each point $y \in B\left(2 R, f_{0}, f_{1}\right)$. Also, there exists a number $\lambda_{1}=\lambda_{1}(R, F, N)>1$ depending only on listed parameters such that for all $\lambda \geq \lambda_{1}$ the functional $J_{\lambda, \alpha}(y)$ is strictly convex on the set $\overline{B\left(R, f_{0}, f_{1}\right)}$, i.e. for all $y_{(1)}, y_{(2)} \in \overline{B\left(R, f_{0}, f_{1}\right)}$

$$
J_{\lambda, \alpha}\left(y_{(2)}\right)-J_{\lambda, \alpha}\left(y_{(1)}\right)-J_{\lambda, \alpha}^{\prime}\left(y_{(1)}\right)\left(y_{(2)}-y_{(1)}\right) \geq C_{1}\left\|y_{(2)}-y_{(1)}\right\|_{H^{2}(0,1)}^{2} .
$$

Corollary 4.1. Consider the functional

$$
\Phi_{\lambda, \alpha}(p)=J_{\lambda, \alpha}(p+f), \quad \forall p \in \overline{B_{0}(R)} .
$$

Then a direct analog of Theorem 4.1 is valid for this functional. More precisely, the functional $\Phi_{\lambda, \alpha}(P)$ has the Frechét derivative $\Phi_{\lambda, \alpha}^{\prime}(p)$ at each point $p \in$ $B_{0}(2 R)$. Let $\lambda_{1}=\lambda_{1}(R, F, N)>1$ be the number of Theorem 4.1. Then there exists a number $\lambda_{2}=\lambda_{2}\left(R, F, N, \chi, f^{*}\right) \geq \lambda_{1}$ depending only on listed parameters such that for all $\lambda \geq \lambda_{2}$ the functional $\Phi_{\lambda, \alpha}(p)$ is strictly convex on the set $\overline{B_{0}(R)}$, i.e. for all $p_{1}, p_{2} \in \overline{B_{0}(R)}$

$$
\Phi_{\lambda, \alpha}\left(p_{2}\right)-\Phi_{\lambda, \alpha}\left(p_{1}\right)-\Phi_{\lambda, \alpha}^{\prime}\left(p_{1}\right)\left(p_{2}-p_{1}\right) \geq C_{2}\left\|p_{2}-p_{1}\right\|_{H^{2}(0,1)}^{2} .
$$

Theorem 4.2. The Frechét derivatives $J_{\lambda, \alpha}^{\prime}(y)$ and $\Phi_{\lambda, \alpha}^{\prime}(P)$ of both functionals $J_{\lambda, \alpha}(y)$ and $\Phi_{\lambda, \alpha}(P)$ are Lipschitz continuous on $B\left(2 R, f_{0}, f_{1}\right)$ and $B_{0}(2 R)$ respectively. In other words, there exists a constant $D=D(R, F, \lambda, \alpha)>$ 0 depending only on listed parameters such that for all $y_{(1)}, y_{(2)} \in B\left(2 R, f_{0}, f_{1}\right)$

$$
\left\|J_{\lambda, \alpha}^{\prime}\left(y_{(1)}\right)-J_{\lambda, \alpha}^{\prime}\left(y_{(2)}\right)\right\|_{H^{2}(0,1)} \leq D\left\|y_{(2)}-y_{(1)}\right\|_{H^{2}(0,1)}
$$

and similarly for $\Phi_{\lambda, \alpha}^{\prime}(P)$.

Theorem 4.3. Let $\lambda_{1}=\lambda_{1}(R, F, N)>1$ and $\lambda_{2}=\lambda_{2}\left(R, F, N, \chi, f^{*}\right) \geq \lambda_{1}$ be the numbers of Theorem 4.1 and Corollary 4.1 respectively. Let $f(x)$ be the function defined in (41), (42). Then for any $\lambda \geq \lambda_{2}$ and for any $\alpha \in$ $(0,1)$ there exists a unique minimizer $y_{\min , \lambda, \alpha}$ of the functional $J_{\lambda, \alpha}(y)$ on the set $\overline{B\left(R, f_{0}, f_{1}\right)}$. In addition, for these values of $\lambda$ and $\alpha$ there exists unique minimizer $p_{\min , \lambda, \alpha}$ of the functional $\Phi_{\lambda, \alpha}(p)$ on the set $\overline{B_{0}(R)}$. Furthermore, $p_{\min , \lambda, \alpha}=y_{\min , \lambda, \alpha}-f$ and

$$
\begin{gathered}
\Phi_{\lambda, \alpha}^{\prime}\left(p_{\min , \lambda, \alpha}\right)\left(p_{\min , \lambda, \alpha}-p\right) \leq 0, \quad \forall p \in \overline{B_{0}(R)}, \\
J_{\lambda, \alpha}^{\prime}\left(y_{\min , \lambda, \alpha}\right)\left(y_{\min , \lambda, \alpha}-y\right) \leq 0, \quad \forall y \in \overline{B\left(R, f_{0}, f_{1}\right)} .
\end{gathered}
$$

Theorem 4.4 (accuracy estimate). Assume that the exact solution $y^{*}$ of the problem (39), (40) exists and $y^{*} \in B\left(R, f_{0}^{*}, f_{1}^{*}\right)$. Also, assume that (46) and (47) hold. Denote $p^{*}=y^{*}-f^{*} \in B_{0}(R)$. In addition, assume that there exists the exact solution $c^{*}(x)$ of our CIP and this function satisfies conditions (1), 
2). Suppose that the function $c^{*}(x)$ can be found from the vector function $y^{*}(x)$ via formula (35) in which functions $y_{n}$ are replaced with components $y_{n}^{*}$ of the vector function $y^{*}(x)$. Let $\lambda_{1}=\lambda_{1}(R, F, N)>1$ and $\lambda_{2}=\lambda_{2}\left(R, F, N, \chi, f^{*}\right) \geq$ $\lambda_{1}$ be the numbers of Theorem 4.1 and Corollary 4.1 respectively. Consider the number $\delta_{0}$ such that $\delta_{0} \in\left(0, e^{-4 \lambda_{2}}\right)$. For any $\delta \in\left(0, \delta_{0}\right)$ we set $\lambda=$ $\lambda(\delta)=\ln \left(\delta^{-1 / 4}\right)>\lambda_{2}$ and $\alpha=\alpha(\delta)=\sqrt{\delta}$. Let $y_{\min , \lambda, \alpha} \in B\left(R, f_{0}, f_{1}\right)$ and $p_{\min , \lambda, \alpha}=y_{\min , \lambda, \alpha}-f \in B_{0}(R)$ be the unique minimizers of the functionals $J_{\lambda, \alpha}(V)$ and $\Phi_{\lambda, \alpha}(p)$ on sets $B\left(R, f_{0}, f_{1}\right)$ and $B_{0}(R)$ respectively (Theorem 4.3). Then the following accuracy estimates hold:

$$
\begin{gathered}
\left\|p_{\min , \lambda, \alpha}-p^{*}\right\|_{H^{2}(0,1)} \leq C_{2} \delta^{1 / 4}, \\
\left\|y_{\min , \lambda(\delta), \alpha(\delta)}-y^{*}\right\|_{H^{2}(0,1)} \leq C_{2} \delta^{1 / 4}, \\
\left\|c_{\min , \lambda(\delta), \alpha(\delta)}-c^{*}\right\|_{L_{2}(0,1)} \leq C_{2} \delta^{1 / 4},
\end{gathered}
$$

where the function $c_{\min , \lambda(\delta), \alpha(\delta)}$ is found from components $y_{n, \min , \lambda(\delta), \alpha(\delta)}$ of the vector function $y_{\min , \lambda(\delta), \alpha(\delta)}$ via formula (35).

Define the subspace $H_{0}^{2}(0,1)$ of the space $H^{2}(0,1)$ as

$$
H_{0}^{2}(0,1)=\left\{w \in H^{2}(0,1): w(0)=w^{\prime}(0)=w^{\prime}(1)=0\right\} .
$$

By $45 B_{0}(R) \subset H_{0}^{2}(0,1)$. Let $Q_{\overline{B_{0}}}: H_{0}^{2}(0,1) \rightarrow \overline{B_{0}(R)}$ be the projection operator of the space $H_{0}^{2}(0,1)$ on the closed ball $\overline{B_{0}(R)}$. Consider now the gradient projection method of the minimization of the functional 52 on the set $\overline{B_{0}(R)}$. Let $p_{0} \in B_{0}(R)$ be an arbitrary point and $\gamma>0$ be a number. We consider the following sequence:

$$
p_{n}=Q_{\overline{B_{0}}}\left(p_{n-1}-\gamma \Phi_{\lambda, \alpha}^{\prime}\left(p_{n-1}\right)\right), \quad n=1,2, \ldots
$$

Theorem 4.5 (global convergence of the gradient method). Let conditions of Theorem 4.4 about exact solutions $y^{*}$ and $c^{*}$ hold. Let the numbers $\lambda_{1}, \lambda_{2}, \delta, \lambda(\delta), \alpha(\delta)$ be the same as in Theorem 4.4. Let an arbitrary point $p_{0} \in B_{0}(R)$ be the starting point of the gradient projection method (58). Consider the sequence (58) and denote $y^{n}=p_{n}+f$. Then there exists a number $\gamma_{0}=\gamma_{0}\left(R, F, N, \chi, f^{*}, \delta\right) \in(0,1)$ depending only on listed parameters such that for any $\gamma \in\left(0, \gamma_{0}\right)$ there exists a number $q=q(\gamma) \in(0,1)$ such that the following convergence estimates are valid:

$$
\begin{gathered}
\left\|p_{n}-p_{\min , \lambda(\delta), \alpha(\delta)}\right\|_{H^{2}(0,1)} \leq q^{n}\left\|p_{0}-p_{\min , \lambda(\delta), \alpha(\delta)}\right\|_{H^{2}(0,1)}, n=1,2, \ldots, \\
\left\|y^{n}-y_{\min , \lambda(\delta), \alpha(\delta)}\right\|_{H^{2}(0,1)} \leq q^{n}\left\|y^{0}-y_{\min , \lambda(\delta), \alpha(\delta)}\right\|_{H^{2}(0,1)}, n=1,2, \ldots \\
\left\|y^{n}-y^{*}\right\|_{H^{2}(0,1)} \leq C_{2} \delta^{1 / 4}+q^{n}\left\|y^{0}-y_{\min , \lambda(\delta), \alpha(\delta)}\right\|_{H^{2}(0,1)}, n=1,2, \ldots \\
\left\|c^{n}-c^{*}\right\|_{L_{2}(0,1)} \leq C_{2} \delta^{1 / 4}+q^{n}\left\|y^{0}-y_{\min , \lambda(\delta), \alpha(\delta)}\right\|_{H^{2}(0,1)}, n=1,2, \ldots
\end{gathered}
$$

where functions $c^{n}(x)$ are found from components $y_{m}^{n}, m=0, \ldots, N-1$ of vector functions $y^{n}$ via formula (35).

Remark 4.1. Since $R>0$ is an arbitrary number and $p_{0}$ is an arbitrary point of the ball $B_{0}(R)$, then Theorem 4.5 claims the global convergence of the gradient projection method (58), see section 1 for our definition of this term. 


\section{Proofs}

In this section we prove theorems formulated in section 4. The proof of Corollary 4.1 is completely similarly with the proof of Theorem 4.1. The same is true about the similarity of the proof of Theorem 4.2 with the proof of Theorem 3.1 of [10]. Hence, we omit proofs of Corollary 4.1 and Theorem 4.2 .

\subsection{Proof of Theorem 4.1}

Below $(,)_{2}$ is the scalar product in $H^{2}(0,1)$. Since we work with complex valued $N-\mathrm{D}$ vector functions, it is convenient to consider them as pairs of real valued functions,

$$
p(x)=(\operatorname{Re} p(x), \operatorname{Im} p(x))=\left(p_{1}(x), p_{2}(x)\right) .
$$

Thus, in fact we work with real valued $N-\mathrm{D}$ vector functions $p_{1}(x), p_{2}(x)$ and the vector function $p(x)$ is $2 N-\mathrm{D}$. We use standard definitions of scalar products for Hilbert spaces of vector functions. Also, for brevity, notations of those spaces of vector functions are the same as ones for regular functions. The use of the complex conjugation below is for convenience only. Below we use the following formula

$$
|a|^{2}-|b|^{2}=(a-b) \bar{a}+(\bar{a}-\bar{b}) b, \quad \forall a, b \in \mathbb{C} .
$$

For complex valued vectors $a=\left(a_{1}, a_{2}\right), b=\left(b_{1}, b_{2}\right)$ with $a_{1}, a_{2}, b_{1}, b_{2} \in \mathbb{R}^{N}$ and with the notation [,] for the scalar product in $\mathbb{R}^{2 N}(63)$ becomes

$$
|a|^{2}-|b|^{2}=[a-b, \bar{a}]+[\bar{a}-\bar{b}, b] .
$$
44

Let vector functions $y_{(1)}, y_{(2)} \in B\left(2 R, f_{0}, f_{1}\right)$. Denote $h=y_{(2)}-y_{(1)}$. By

$$
\begin{gathered}
h(0)=h^{\prime}(0)=h^{\prime}(1)=0, \\
\|h\|_{H^{2}(0,1)} \leq 4 R .
\end{gathered}
$$

In particular, 65 implies that $h \in H_{0}^{2}(0,1)$. Also, by embedding theorem $H^{2}(0,1) \subset C^{1}[0,1]$. Hence, 66 implies that with a generic constant $C$,

$$
\left\|h^{\prime}\right\|_{C[0,1]} \leq C R
$$

Keeping in mind of using (64), denote

$$
a=\left(y_{(1)}+h\right)^{\prime \prime}+F\left(y_{(1)}^{\prime}+h^{\prime}\right), b=y_{(1)}^{\prime \prime}+F\left(y_{(1)}^{\prime}\right)
$$

Hence,

$$
a-b=h^{\prime \prime}+F\left(y_{(1)}^{\prime}+h^{\prime}\right)-F\left(y_{(1)}^{\prime}\right) .
$$

Recalling that the vector function $F\left(y^{\prime}\right)$ is quadratic with respect to the components $y_{n}^{\prime}(x)$ of the vector function $y^{\prime}$, we obtain

$$
\begin{gathered}
F\left(y_{(1)}^{\prime}+h^{\prime}\right)-F\left(y_{(1)}^{\prime}\right)=G_{1}\left(y_{(1)}^{\prime}, h^{\prime}\right)+G_{2}\left(y_{(1)}^{\prime}, h^{\prime}\right) \\
a-b=h^{\prime \prime}+G_{1}\left(y_{(1)}^{\prime}, h^{\prime}\right)+G_{2}\left(y_{(1)}^{\prime}, h^{\prime}\right)
\end{gathered}
$$


where the vector function $G_{1}\left(y_{(1)}^{\prime}, h^{\prime}\right)$ is linear with respect to each $y_{(1)}^{\prime}$ and $h^{\prime}$. The following estimates are valid:

$$
\begin{gathered}
\left|G_{1}\left(y_{(1)}^{\prime}, h^{\prime}\right)\right| \leq C_{1}\left|h^{\prime}\right|, \\
\left|G_{2}\left(y_{(1)}^{\prime}, h^{\prime}\right)\right| \leq C_{1}\left|h^{\prime}\right|^{2} .
\end{gathered}
$$

Hence, keeping in mind 64, 68 -670), we obtain

$$
\begin{gathered}
{[a-b, \bar{a}]=} \\
{\left[h^{\prime \prime}+G_{1}\left(y_{(1)}^{\prime}, h^{\prime}\right)+G_{2}\left(y_{(1)}^{\prime}, h^{\prime}\right), \overline{\left(y_{(1)}+h\right)^{\prime \prime}+F\left(y_{(1)}^{\prime}+h^{\prime}\right)}\right]} \\
=\left[h^{\prime \prime}+G_{1}\left(y_{(1)}^{\prime}, h^{\prime}\right), \overline{\left.y_{(1)}^{\prime \prime}+F\left(y_{(1)}^{\prime}\right)\right]+\left|h^{\prime \prime}\right|^{2}}\right. \\
+\left[h^{\prime \prime}, \overline{G_{1}\left(y_{(1)}^{\prime}, h^{\prime}\right)+G_{2}\left(y_{(1)}^{\prime}, h^{\prime}\right)}\right]+\left[G_{1}\left(y_{(1)}^{\prime}, h^{\prime}\right)+G_{2}\left(y_{(1)}^{\prime}, h^{\prime}\right), \overline{h^{\prime \prime}}\right] \\
+\left[G_{1}\left(y_{(1)}^{\prime}, h^{\prime}\right), \overline{G_{2}\left(y_{(1)}^{\prime}, h^{\prime}\right)}\right]+\left[G_{2}\left(y_{(1)}^{\prime}, h^{\prime}\right), \overline{G_{1}\left(y_{(1)}^{\prime}, h^{\prime}\right)}\right] \\
+\left|G_{1}\left(y_{(1)}^{\prime}, h^{\prime}\right)\right|^{2}+\left|G_{2}\left(y_{(1)}^{\prime}, h^{\prime}\right)\right|^{2}+\left[G_{2}\left(y_{(1)}^{\prime}, h^{\prime}\right), \overline{y_{(1)}^{\prime \prime}+F\left(y_{(1)}^{\prime}\right)}\right]
\end{gathered}
$$

Let $S_{1}(x)$ denotes the sum of 3rd, 4th and 5th lines of 73 ,

$$
\begin{gathered}
S_{1}(x)=\left|h^{\prime \prime}\right|^{2}+\left[h^{\prime \prime}, \overline{G_{1}\left(y_{(1)}^{\prime}, h^{\prime}\right)+G_{2}\left(y_{(1)}^{\prime}, h^{\prime}\right)}\right] \\
+\left[G_{1}\left(y_{(1)}^{\prime}, h^{\prime}\right)+G_{2}\left(y_{(1)}^{\prime}, h^{\prime}\right) \overline{h^{\prime \prime}}\right]+\left[G_{1}\left(y_{(1)}^{\prime}, h^{\prime}\right), \overline{G_{2}\left(y_{(1)}^{\prime}, h^{\prime}\right)}\right] \\
+\left[G_{2}\left(y_{(1)}^{\prime}, h^{\prime}\right), \overline{G_{1}\left(y_{(1)}^{\prime}, h^{\prime}\right)}\right]+\left|G_{1}\left(y_{(1)}^{\prime}, h^{\prime}\right)\right|^{2}+\left|G_{2}\left(y_{(1)}^{\prime}, h^{\prime}\right)\right|^{2} \\
+\left[G_{2}\left(y_{(1)}^{\prime}, h^{\prime}\right), \overline{y_{(1)}^{\prime \prime}+F\left(y_{(1)}^{\prime}\right)}\right] .
\end{gathered}
$$

Then, using the inequality

$$
\left[d_{1}, d_{2}\right] \geq-\frac{1}{4}\left|d_{1}\right|^{2}-\left|d_{2}\right|^{2}, \quad \forall d_{1}, d_{2} \in \mathbb{R}^{2 N},
$$

(67), 71) and (72), we obtain the following estimate from the below for $S_{1}(x)$,

$$
S_{1}(x) \geq \frac{1}{2}\left|h^{\prime \prime}(x)\right|^{2}-C_{1}\left|h^{\prime}(x)\right|^{2}, \quad x \in(0,1) .
$$

By $(73)[a-b, \bar{a}]$ can be written as

$$
[a-b, \bar{a}]=\left[h^{\prime \prime}+G_{1}\left(y_{(1)}^{\prime}, h^{\prime}\right), \overline{y_{(1)}^{\prime \prime}+F\left(y_{(1)}^{\prime}\right)}\right]+S_{1} .
$$


Similarly the second term in the right hand side of 64 can be represented as

$$
\begin{aligned}
{[\bar{a}-\bar{b}, b]=\left[\overline{h^{\prime \prime}+G_{1}\left(y_{(1)}^{\prime}, h^{\prime}\right)+G_{2}\left(y_{(1)}^{\prime}, h^{\prime}\right)}, y_{(1)}^{\prime \prime}+F\left(y_{(1)}^{\prime}\right)\right] } \\
=\left[\overline{h^{\prime \prime}+G_{1}\left(y_{(1)}^{\prime}, h^{\prime}\right)}, y_{(1)}^{\prime \prime}+F\left(y_{(1)}^{\prime}\right)\right] \\
+\left[\overline{G_{2}\left(y_{(1)}^{\prime}, h^{\prime}\right)}, y_{(1)}^{\prime \prime}+F\left(y_{(1)}^{\prime}\right)\right]
\end{aligned}
$$

where by 72

$$
\left[\overline{G_{2}\left(y_{(1)}^{\prime}, h^{\prime}\right)}, y_{(1)}^{\prime \prime}+F\left(y_{(1)}^{\prime}\right)\right]=S_{2}(x) \geq-C_{1}\left|h^{\prime}\right|^{2} .
$$

Thus, (63), (68) and (73)- 78 imply that

$$
|a|^{2}-|b|^{2}=\operatorname{Lin}(h)+S(x),
$$

where the term $\operatorname{Lin}(h)$ is linear with respect to the vector function $h=\left(h_{1}, h_{2}\right)$,

$$
\begin{gathered}
\operatorname{Lin}(h)=\left[h^{\prime \prime}+G_{1}\left(y_{(1)}^{\prime}, h^{\prime}\right), \overline{y_{(1)}^{\prime \prime}+F\left(y_{(1)}^{\prime}\right)}\right] \\
\left.+\overline{h^{\prime \prime}+G_{1}\left(y_{(1)}^{\prime}, h^{\prime}\right)}, y_{1}^{\prime \prime}+F\left(y_{(1)}^{\prime}\right)\right]
\end{gathered}
$$

and the function $S(x)$ is

$$
S(x)=S_{1}(x)+S_{2}(x) \geq \frac{1}{2}\left|h^{\prime \prime}(x)\right|^{2}-C_{1}\left|h^{\prime}(x)\right|^{2} .
$$

Next, it follows from $72,773,(74)$ and $(78)$ that

$$
|S(x)| \leq C_{1}\left(\left|h^{\prime \prime}\right|^{2}+\left|h^{\prime}\right|^{2}\right)(x) \text {. }
$$

Hence, (50) implies that

$$
\begin{gathered}
J_{\lambda, \alpha}\left(y_{(1)}+h\right)-J_{\lambda, \alpha}\left(y_{(1)}\right) \\
=e^{2 \lambda} \int_{0}^{1} \operatorname{Lin}(h) e^{-2 \lambda x} d x+\alpha\left(h, y_{(1)}\right)_{2}+\alpha\left(y_{(1)}, h\right)_{2} \\
+e^{2 \lambda} \int_{0}^{1} S(x) e^{-2 \lambda x} d x+\alpha\|h\|_{H^{2}(0,1)}^{2} .
\end{gathered}
$$

Let $L_{\lambda, \alpha}(h)$ denotes the second line of $(82)$. Then $(65),(71)$ and $(79)$ imply that $L_{\lambda, \alpha}(h): H_{0}^{2}(0,1) \rightarrow \mathbb{R}$ is a bounded linear functional. Hence, by Riesz theorem, there exists an element $w_{\lambda, \alpha} \in H_{0}^{2}(0,1)$ such that

$$
L_{\lambda, \alpha}(h)=\left(w_{\lambda, \alpha}, h\right)_{2}, \quad \forall h=\left(h_{1}, h_{2}\right) \in H_{0}^{2}(0,1) .
$$


Hence, 81), 82 and 83 imply that

$$
J_{\lambda, \alpha}\left(y_{(1)}+h\right)-J_{\lambda, \alpha}\left(y_{(1)}\right)=\left(w_{\lambda, \alpha}, h\right)_{2}+o\left(\|h\|_{H^{2}(0,1)}\right)
$$

as $\|h\|_{H^{2}(0,1)} \rightarrow 0$. Hence, $w_{\lambda, \alpha} \in H_{0}^{2}(0,1)$ is the Frechét derivative $J_{\lambda, \alpha}^{\prime}\left(y_{(1)}\right)$ of the functional $J_{\lambda, \alpha}$ at the point $y_{(1)}$, i.e. $J_{\lambda, \alpha}^{\prime}\left(y_{(1)}\right)=w_{\lambda, \alpha}$. Thus, the existence of the Frechét derivative of the functional $J_{\lambda, \alpha}(y)$ at any point $y \in B\left(2 R, f_{0}, f_{1}\right)$ is established.

Below we prove the strict convexity estimate 51 . Let now $y_{(1)} \in \overline{B\left(R, f_{0}, f_{1}\right)}$ and $y_{(2)} \in \overline{B\left(R, f_{0}, f_{1}\right)}$ be two arbitrary points. We keep the same notation for their difference $h=y_{(1)}-y_{(2)}$. The vector function $h$ satisfies boundary conditions 65, and in (66) $4 R$ should be replaced with $2 R$. Thus, by 80 and 82

$$
\begin{gathered}
J_{\lambda, \alpha}\left(y_{(1)}+h\right)-J_{\lambda, \alpha}\left(y_{(1)}\right)-J_{\lambda, \alpha}^{\prime}\left(y_{(1)}\right)(h) \\
=e^{2 \lambda} \int_{0}^{1} S(x) e^{-2 \lambda x} d x+\alpha\|h\|_{H^{2}(0,1)}^{2} \geq \frac{1}{2} e^{2 \lambda} \int_{0}^{1}\left|h^{\prime \prime}\right|^{2} e^{-2 \lambda x} d x \\
-C_{1} e^{2 \lambda} \int_{0}^{1}\left|h^{\prime}\right|^{2} e^{-2 \lambda x} d x+\alpha\|h\|_{H^{2}(0,1)}^{2} \cdot
\end{gathered}
$$

Using Lemma 3.2 and boundary conditions 65 , we estimate from the below the second line of 85 as

$$
\begin{gathered}
\frac{1}{2} e^{2 \lambda} \int_{0}^{1}\left|h^{\prime \prime}\right|^{2} e^{-2 \lambda x} d x-C_{1} e^{2 \lambda} \int_{0}^{1}\left|h^{\prime}\right|^{2} e^{-2 \lambda x} d x+\alpha\|h\|_{H^{2}(0,1)}^{2} \\
\geq C e^{2 \lambda} \int_{0}^{1}\left|h^{\prime \prime}\right|^{2} e^{-2 \lambda x} d x+C \lambda e^{2 \lambda} \int_{0}^{1}\left|h^{\prime}\right|^{2} e^{-2 \lambda x} d x \\
+C \lambda^{3} e^{2 \lambda} \int_{0}^{1}|h|^{2} e^{-2 \lambda x} d x-C_{1} e^{2 \lambda} \int_{0}^{1}\left|h^{\prime}\right|^{2} e^{-2 \lambda x} d x+\alpha\|h\|_{H^{2}(0,1)}^{2}, \forall \lambda \geq 1 .
\end{gathered}
$$

Hence, one can choose a sufficiently large number $\lambda_{1}=\lambda_{1}(R, F, N)>1$ such that for all $\lambda \geq \lambda_{1}$ we have $C_{1}<C \lambda / 2$. Note that $e^{2 \lambda} e^{-2 \lambda x}>1$ for $x \in(0,1)$. Hence, (85) and (86) imply

$$
\begin{gathered}
J_{\lambda, \alpha}\left(y_{(1)}+h\right)-J_{\lambda, \alpha}\left(y_{(1)}\right)-J_{\lambda, \alpha}^{\prime}\left(y_{(1)}\right)(h) \\
\geq \frac{1}{2} e^{2 \lambda} \int_{0}^{1}\left|h^{\prime \prime}\right|^{2} e^{-2 \lambda x} d x-C_{1} e^{2 \lambda} \int_{0}^{1}\left|h^{\prime}\right|^{2} e^{-2 \lambda x} d x+\alpha\|h\|_{H^{2}(0,1)}^{2} \\
\geq C e^{2 \lambda} \int_{0}^{1}\left|h^{\prime \prime}\right|^{2} e^{-2 \lambda x} d x+C_{1} \lambda e^{2 \lambda} \int_{0}^{1}\left|h^{\prime}\right|^{2} e^{-2 \lambda x} d x
\end{gathered}
$$




$$
\begin{gathered}
+C_{1} \lambda^{3} e^{2 \lambda} \int_{0}^{1}|h|^{2} e^{-2 \lambda x} d x+\alpha\|h\|_{H^{2}(0,1)}^{2} \\
\geq C_{1} \int_{0}^{1}\left(\left|h^{\prime \prime}\right|^{2}+\left|h^{\prime}\right|^{2}+|h|^{2}\right) d x+\alpha\|h\|_{H^{2}(0,1)}^{2} \\
=\left(C_{1}+\alpha\right)\|h\|_{H^{2}(0,1)}^{2} \geq C_{1}\|h\|_{H^{2}(0,1)}^{2},
\end{gathered}
$$

which is equivalent with (51).

It is important for the proof of Theorem 4.4 to point out that, until (83), we have not used boundary conditions 65 for the vector function $h(x)$. Hence, 171, 79, ,81 and 82 imply that

$$
\begin{gathered}
\left|J_{\lambda, \alpha}(y+z)-J_{\lambda, \alpha}(y)\right| \leq C_{1}\|z\|_{H^{2}(0,1)} e^{2 \lambda}, \\
\forall y \in B\left(2 R, f_{0}, f_{1}\right), \quad \forall z \in\left\{z \in H^{2}(0,1):\|z\|_{H^{2}(0,1)}<4 R\right\} .
\end{gathered}
$$

\subsection{Proof of Theorem 4.3}

Let $f(x) \in C^{2}[0,1]$ be the function defined in 41 , 42,. Then Proposition 3.1 implies that

$$
p=y-f \in \overline{B_{0}(R)}, \quad \forall y \in \overline{B\left(R, f_{0}, f_{1}\right)} .
$$

It follows immediately from Theorem 2.1 of 10 and Corollary 4.1 that for any $\lambda \geq \lambda_{2}$ there exists unique minimizer $p_{\min , \lambda, \alpha}$ of the functional $\Phi_{\lambda, \alpha}(p)$ on the set $\overline{B_{0}(R)}$,

$$
\min _{p \in \overline{B_{0}(R)}} \Phi_{\lambda, \alpha}(p)=\Phi_{\lambda, \alpha}\left(p_{\min , \lambda, \alpha}\right)
$$

Consider now the vector function $\widetilde{y}_{\lambda, \alpha}$,

$$
\widetilde{y}_{\lambda, \alpha}(x)=p_{\min , \lambda, \alpha}(x)+f(x) .
$$

By Proposition 3.1 the function $\widetilde{y}_{\lambda, \alpha} \in \overline{B\left(R, f_{0}, f_{1}\right)}$. Let $y \neq \widetilde{y}_{\lambda, \alpha}$ be an arbitrary vector function from $\overline{B\left(R, f_{0}, f_{1}\right)}$. Denote $p_{y}=y-f$. Then by Proposition 3.1 $p_{y} \in \overline{B_{0}(R)}$. We have:

$$
J_{\lambda, \alpha}(y)=J_{\lambda, \alpha}((y-f)+f)=J_{\lambda, \alpha}\left(p_{y}+f\right) .
$$

And by 52

$$
J_{\lambda, \alpha}\left(p_{y}+f\right)=\Phi_{\lambda, \alpha}\left(p_{y}\right) .
$$

Since $p_{y} \neq p_{\min , \lambda, \alpha}$, then

$$
\Phi_{\lambda, \alpha}\left(p_{y}\right)>\Phi_{\lambda, \alpha}\left(p_{\min , \lambda, \alpha}\right) .
$$

On the other hand, by (52) and 91 - 94

$$
J_{\lambda, \alpha}(y)=\Phi_{\lambda, \alpha}\left(p_{y}\right)>\Phi_{\lambda, \alpha}\left(p_{\min , \lambda, \alpha}\right)=J_{\lambda, \alpha}\left(\widetilde{y}_{\lambda, \alpha}\right) .
$$

Hence, 95 implies that $\widetilde{y}_{\lambda, \alpha}$ is the unique minimizer $y_{\min , \lambda, \alpha}$ of the functional $J_{\lambda, \alpha}$ on the set $\overline{B\left(R, f_{0}, f_{1}\right)}$,

$$
\frac{\min }{B\left(R, f_{0}, f_{1}\right)} J_{\lambda, \alpha}(y)=J_{\lambda, \alpha}\left(y_{\min , \lambda, \alpha}\right) .
$$

Inequalities (53) and (54) follow immediately from Lemma 2.1 of [10, 50 and (96) respectively. 


\subsection{Proof of Theorem 4.4}

Since $\left(y^{*}\right)^{\prime \prime}+F\left(\left(y^{*}\right)^{\prime}\right)=0$ and $\alpha=\sqrt{\delta}$, then 50 implies that

$$
J_{\lambda, \alpha}\left(y^{*}\right)=\alpha\left\|y^{*}\right\|_{H^{2}(0,1)}^{2} \leq \sqrt{\delta} R^{2} .
$$

Recall that

$$
y^{*}-f^{*}=p^{*} \in B_{0}(R) .
$$

We have

$$
J_{\lambda, \alpha}\left(p^{*}+f\right)=J_{\lambda, \alpha}\left(\left(p^{*}+f^{*}\right)+\left(f-f^{*}\right)\right)=J_{\lambda, \alpha}\left(y^{*}+\left(f-f^{*}\right)\right) .
$$

Temporary denote $h=f-f^{*}$. Since by Proposition $3.1\left(p^{*}+f\right) \in B\left(R, f_{0}, f_{1}\right)$, then, using (49), 87), (88), (97) and (98), we obtain

$$
J_{\lambda, \alpha}\left(p^{*}+f\right)=J_{\lambda, \alpha}\left(y^{*}+h\right) \leq J_{\lambda, \alpha}\left(y^{*}\right)+B \delta e^{2 \lambda} \leq C_{2}\left(\sqrt{\delta}+\delta e^{2 \lambda}\right),
$$

see the line below 49 for the definition of the number $B=B(\chi)>0$. Choose a number $\delta_{0} \in\left(0, e^{-4 \lambda_{2}}\right)$. Let $\delta \in\left(0, \delta_{0}\right)$. Choose $\lambda=\lambda(\delta)=\ln \left(\delta^{-1 / 4}\right)$. Then $\lambda(\delta)>\lambda_{2}$ and $\delta e^{2 \lambda}=\sqrt{\delta}$. Hence, 100 implies that

$$
J_{\lambda, \alpha}\left(p^{*}+f\right) \leq C_{2} \sqrt{\delta}
$$

Let

By 52

$$
p_{\min , \lambda, \alpha}=y_{\min , \lambda, \alpha}-f \text {. }
$$

$$
J_{\lambda, \alpha}\left(p^{*}+f\right)-J_{\lambda, \alpha}\left(p_{\min , \lambda, \alpha}+f\right)=\Phi_{\lambda, \alpha}\left(p^{*}\right)-\Phi_{\lambda, \alpha}\left(p_{\min , \lambda, \alpha}\right) .
$$

Hence, by Corollary 4.1,

$$
\begin{gathered}
J_{\lambda, \alpha}\left(p^{*}+f\right)-J_{\lambda, \alpha}\left(p_{\min , \lambda, \alpha}+f\right) \\
-J_{\lambda, \alpha}^{\prime}\left(p_{\min , \lambda, \alpha}+f\right)\left(\left(p^{*}+f\right)-\left(p_{\min , \lambda, \alpha}+f\right)\right) \\
=\Phi_{\lambda, \alpha}\left(p^{*}\right)-\Phi_{\lambda, \alpha}\left(p_{\min , \lambda, \alpha}\right)-\Phi_{\lambda, \alpha}^{\prime}\left(p_{\min , \lambda, \alpha}\right)\left(p^{*}-p_{\min , \lambda, \alpha}\right) \\
\geq C_{2}\left\|p^{*}-p_{\min , \lambda, \alpha}\right\|_{H^{2}(0,1)}^{2} .
\end{gathered}
$$

Next, by $53-\Phi_{\lambda, \alpha}^{\prime}\left(p_{\min , \lambda, \alpha}\right)\left(p^{*}-p_{\min , \lambda, \alpha}\right) \leq 0$. Hence, 103 implies that

$$
\Phi_{\lambda, \alpha}\left(p^{*}\right) \geq C_{2}\left\|p^{*}-p_{\min , \lambda, \alpha}\right\|_{H^{2}(0,1)}^{2} .
$$

On the other hand, since by 52$) \Phi_{\lambda, \alpha}\left(p^{*}\right)=J_{\lambda, \alpha}\left(p^{*}+f\right)$, then, using 101) and (104), we obtain

$$
\left\|p^{*}-p_{\min , \lambda, \alpha}\right\|_{H^{2}(0,1)} \leq C_{2} \delta^{1 / 4}
$$

which establishes (55). Next,

$$
\begin{gathered}
\left\|p^{*}-p_{\min , \lambda, \alpha}\right\|_{H^{2}(0,1)}=\left\|\left(p^{*}+f^{*}\right)-\left(p_{\min , \lambda, \alpha}+f\right)+\left(f-f^{*}\right)\right\|_{H^{2}(0,1)} \\
\geq\left\|y^{*}-y_{\min , \lambda, \alpha}\right\|_{H^{2}(0,1)}-\left\|f-f^{*}\right\|_{H^{2}(0,1)} \\
\geq\left\|y^{*}-y_{\min , \lambda, \alpha}\right\|_{H^{2}(0,1)}-B \delta .
\end{gathered}
$$

Here, to obtain the term $B \delta$, we have used (49). Hence, using (55) and (105), we obtain $\left\|y^{*}-y_{\min , \lambda, \alpha}\right\|_{H^{2}(0,1)} \leq C_{2} \delta^{1 / 4}$, which is the same as estimate (56). Estimate (57) follows immediately from (35) and (56). 


\subsection{Proof of Theorem 4.5}

Estimate 59 follows immediately from the combination of Corollary 4.1 and Theorem 4.3 with Theorem 2.1 of [10]. Given that $y^{n}=p_{n}+f$ and also that by Theorem $4.3 y_{\min , \lambda, \alpha}=p_{\min , \lambda, \alpha}+f,(59)$ implies $(60)$. Next, by triangle inequality

$$
\left\|y^{n}-y^{*}\right\|_{H^{2}(0,1)} \leq\left\|y_{\min , \lambda(\delta), \alpha(\delta)}-y^{*}\right\|_{H^{2}(0,1)}+\left\|y^{n}-y_{\min , \lambda(\delta), \alpha(\delta)}\right\|_{H^{2}(0,1)} .
$$

Hence, estimate (61) follows from (56), 60 and (106). Finally, 62 follows from (35), (56) and (61).

\section{$6 \quad$ Numerical studies}

In this section we present a numerical verification of proposed method by reconstructing the coefficient $c(x)$ from both computationally simulated and experimental data. Since in our experiments we have to image only a single target for each data set, then we focus in our computationally simulated data on the case of a single inclusion only.

\subsection{Data generation}

To generate computationally simulated data for our CIP, we solve the forward problem (3), (4) via solving the one dimensional Lippmann-Schwinger equation (16) in which the function $c(x)$ is set as follows:

$$
c(x):=c_{\text {true }}(x)=\left\{\begin{array}{cc}
\widehat{c}_{\text {true }}, & x \in\left(x_{l o c}-d / 2, x_{l o c}+d / 2\right), \\
1, & \text { elsewhere }
\end{array}\right.
$$

Here, $\widehat{c}_{\text {true }}=$ const. $>0$ is the dielectric constant of a simulated target, $x_{l o c}$ is the location of the center of this target, and $d$ is its width. Thus, targets used in computational simulations are step-wise functions. In numerical experiments we consider the following sets of dielectric constants $\widehat{c}_{\text {true }}$ and locations $x_{\text {loc }}$ :

$$
\widehat{c}_{\text {true }}=\{3.0,4.0,5.0,6.0\}, \quad x_{l o c}=\{0.1,0.2,0.3,0.4\} .
$$

For this study we use the same width $d=0.1$ in 107 for all targets. Since we test four locations of centers for each of these four values of $\widehat{c}_{\text {true }}$, then we reconstruct total sixteen (16) targets.

We generate our simulated data for the interval of wave numbers $k_{m} \in$ $[0.5,1.5]$. This interval is divided into $N_{k}$ equal subintervals. Hence, we obtain a grid of equally spaced points: $k_{m}=\underline{k}+m h_{k}, m=0, \ldots, N_{k}$, where $h_{k}=$ $(\bar{k}-\underline{k}) / N_{k}$. Hence, $k_{0}=\underline{k}=0.5$ and $k_{N_{k}}=\bar{k}=1.5$.

By solving the Lippmann-Schwinger equation (16) for every point $k_{m}$ and taking the values of $u\left(0, k_{m}\right)$ we obtain the noiseless boundary function $g_{0}\left(k_{m}\right)$ in (6). Next, we add the random noise in this function

$$
g_{0, \delta}\left(k_{m}\right)=g_{0}\left(k_{m}\right)\left(1.0+\delta \sigma\left(k_{m}\right)\right), \sigma=\sigma_{r}\left(k_{m}\right)+i \sigma_{r}\left(k_{m}\right), m=0, \ldots N_{k},
$$

where $\delta$ is the noise level, $\sigma_{r}$ and $\sigma_{i}$ are random floating point numbers, uniformly distributed between -1.0 and 1.0. In our computations we use $\delta=0.05$, i.e. our data have $5 \%$ of noise. To reduce this noise the function $g_{0, \delta}\left(k_{n}\right)$ is smoothed out by using the standard averaging procedure. Next, we calculate the boundary function $q_{0, \delta}$ and its derivative $q_{1, \delta}(31)$. 


\subsection{Location estimation and data propagation}

We have discovered in our computations that prior the minimization of the functional (50), it is important to estimate the location of the target first. The same observation was made in 9] for the 3-D case: see Figure 2 in [9]. Procedures of [9] for these estimates are different from the one described here, both of them are heuristic ones. The procedure described below is also a heuristic one.

First, we recall one of steps of [1. Let $v(x, k)$ be the function defined in (28). Rather than considering the truncated Fourier series (34), one can denote in (33) $s(x, k)=v_{k}(x, k)$. Hence,

$$
v(x, k)=-\int_{k}^{\bar{k}} s(x, \tau) d \tau+v(x, \bar{k}) .
$$

Then substitution of 110 in equation 33 results in a nonlinear integro differential equation with respect to the function $s(x, k)$. The function $v(x, \bar{k})$ is also unknown in this equation. In [1] this function is called the "tail function". It follows from $20-22$ that

$$
v(x, k)=\frac{r(x)}{k}+O\left(\frac{\ln (2 k)}{k^{2}}\right), k \rightarrow \infty .
$$

Assuming that the number $\bar{k}>>1$ is sufficiently large, dropping the second term in the right hand side of $(111)$, substituting $v(x, \bar{k})=r(x) / \bar{k}$ in that integro differential equation and setting in it $k:=\bar{k}$, one obtains that $r^{\prime \prime}(x)=0$. Solution of the latter equation for $x \in(0,1)$ with certain boundary conditions at $x=0,1$ provides an approximation for the tail function.

Unlike [1, to estimate the location of the target from our computationally simulated data, we do not assume that $\bar{k}$ is sufficiently large. Nevertheless, we solve the same equation $r^{\prime \prime}(x)=0$. More precisely, we solve the following boundary value problem:

$$
\begin{gathered}
r^{\prime \prime}=0, \quad x \in(0,1), \\
r(0)=q_{0, \delta}(\bar{k}), \quad r^{\prime}(0)=q_{1, \delta}(\bar{k}), \quad r^{\prime}(1)=0 .
\end{gathered}
$$

Since the problem 112, 113 is overdetermined, we solve it by the quasireversibility method (QRM). We refer to 34 for details about this specific version of QRM. More precisely, we minimize the following functional $I_{\alpha}(r)$ :

$$
I_{\gamma}(r)=\frac{1}{2}\left(\left\|r^{\prime \prime}\right\|_{L^{2}(0,1)}+\gamma\|r\|_{L^{2}(0,1)}\right)
$$

subject to boundary conditions 113 . Here $\gamma>0$ is the regularization parameter. Figure 1 depicts the so computed function $r(x)$ for the target with $\widehat{c}_{\text {true }}=5.0$ and $x_{l o c}=0.4$, see $(107)$. Note that the minimal value of $\operatorname{Im} r(x)$ is near the point $x=0.4$, which is the center $x_{l o c}$ of this simulated target. Therefore, we have estimated the location of the center of this target $x_{\text {est }}$. We have seen in all 16 our computational experiments that this approach always works: it gives us the value of $x_{e s t}$ with an error of about $5 \%$.

Next, in cases when the estimated location $x_{e s t}>0.1$, we propagate the data function $g_{0, \delta}(k)$ from the point $x=0.0$, where this function is given, to 


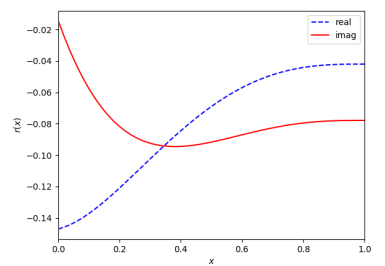

Figure 1: The real (dashed line) and imaginary (solid line) parts of the function $r(x)$ for the target with $\widehat{c}_{\text {true }}=5.0$ and $x_{l o c}=0.4$

the point $x_{t a r}=x_{e s t}-0.1$. By (107) this means that the maximal "allowable" width of the target in our case is 0.2 .

We point out that a 3-D version of the data propagation procedure has been widely used in the previous works of Klibanov with coauthors for both computationally simulated and experimental data, see, e.g. 9, 18, and references cited therein. Since $c(x)=1.0$ for $x \in\left(0, x_{t a r}\right)$ and $x_{0}<0$, then by (3) $u^{\prime \prime}+k^{2} u=0$ for $x \in\left(0, x_{t a r}\right)$. Hence,

$$
u(x, k)=D_{1}(k) e^{i k x}+D_{2}(k) e^{-i k x}, x \in\left(0, x_{t a r}\right),
$$

where complex numbers $D_{1}(k), D_{2}(k)$ depend only on $k$. To find these numbers, we need to know $u(0, k)$ and $u^{\prime}(0, k)$. The latter numbers, in turn can be easily found from formulae (5)-(9). Hence, we perform the data propagation via the following formulae:

$$
\begin{gathered}
g_{\text {prop }}\left(x_{t a r}, k\right)=\frac{u_{p}\left(x_{t a r}, k\right)}{u_{0}\left(x_{t a r}, k\right)}, \quad u_{\text {prop }}\left(x_{t a r}, k\right)=D_{1} e^{i k x_{t a r}}+D_{2} e^{-i k x_{t a r}}, \\
D_{1}=u_{0}(0)\left(g_{0}-1.0\right), \quad D_{2}=u_{0}(0),
\end{gathered}
$$

where $k$-dependent functions $g_{\text {prop }}\left(x_{t a r}, k\right)$ and $u_{\text {prop }}\left(x_{t a r}, k\right)$ play now the role of functions $g_{0}(k)$ and $u(0, k)$ in which $\{x=0\}$ is replaced with $\left\{x=x_{\text {tar }}\right\}$.

\subsection{The optimal number $N$ terms in the expansion (34)}

We need to determine the optimal number $N$ of terms in the truncated Fourier series (34). To do this, we first solve the Lippmann-Schwinger equation 16 for a reference target with $c(x):=\widehat{c}_{\text {true }}(x)$, see $(107)$ for $\widehat{c}_{\text {true }}$. This way we obtain the functions $w_{\text {true }}$ in $(7)$ and $v_{\text {true }}(x, k)$ in (28). Next, we compute vector functions $y_{\text {true }, N}(x)$ in $(34),(37)$ for different values of $N$ and reconstruct approximate functions $c_{a p p r, N}(x)$ via (35). Figure 2 a shows the functions $c_{a p p r, N}(x)$ for $N=1,2,3,4$, where $\widehat{c}_{\text {true }}=5.0$ and $x_{l o c}=0.4$. The corresponding basis functions $\psi_{n}(k)$ are shown on Figure $2 \mathrm{~b}$. One can see that the functions $c_{t r u e, N}(x)$ are accurately approximated for both $N=3$ and $N=4$, and their approximation errors $\varepsilon_{N}=\left\|c_{a p p r, N}-c_{\text {true }}\right\|_{L^{2}(0,1)}$ are sufficiently small: $\varepsilon_{N}=0.07$ and 0.01 , respectively. Therefore, we choose the optimal number of functions in our basis $N=3$. 


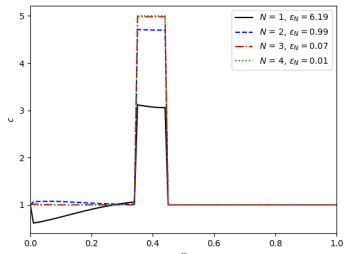

(a)

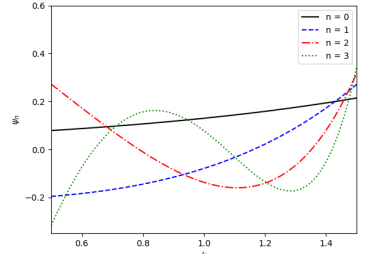

(b)

Figure 2: a) The approximate functions $\left.c_{a p p r, N}(x), b\right)$ basis functions $\phi_{n}(k)$

\subsection{Numerical implementation}

For the numerical solution we use the finite difference discretization method. So we divide the interval $x \in[0,1]$ into $N_{x}$ equal subintervals and obtain the mesh $x_{j}=j h_{x}, j=0, \ldots, N_{x}, h_{x}=1.0 / N_{x}$. Combining this mesh with the mesh for wave numbers $k_{m}$ defined in section 6.1 , we obtain the $N_{k} \times N_{x}$ two dimensional mesh with the grid points $\left(k_{m}, x_{j}\right)$. We need to find the discrete function $V=\left\{v_{m, j}\right\}$, where $v_{m, j}=v\left(k_{m}, x_{j}\right)$ are its values at those grid points. The discrete version of equation (34) can be written as

$$
V=\Psi Y
$$

where $\Psi=\left\{\psi_{m, n}\right\}$ is the $N_{k} \times N$ matrix with $\psi_{m, n}=\psi_{n}\left(k_{m}\right)$ and $Y=\left\{y_{n, j}\right\}$ is the $N \times N_{x}$ two dimensional discrete vector function $y(37)$.

The main objective of the proposed method is to find the minimizer of functional $J_{\lambda, \alpha}(y)$ (50). Naturally, we minimize this functional numerically for the case of the discrete function $Y$. The details of finite difference discretization of the functional and its gradient are not described here for brevity. We refer to [37, where discretization of similar functional is performed analytically using the Kronecker delta function. Although, our theory suggest the gradient projection method for the minimization, we have noticed that the conjugate gradient method (CG) works well for our problem. This method is easier to implement numerically and it gives practically the same results as the gradient projection method. The latter has been consistently observed in the previous works on the numerical issues of the convexification method [1, 8, 9, 12, 21, 36. Also, to decrease the necessity of calculating the functional and its gradient on each iterative step, we have decided not to use the standard line search algorithm to seek the step size of the minimization process. Instead, we begin with the initial step size $10^{-7}$. On each iteration, the step size is reduced 10 times for the next iteration if the value of the functional on the current iteration is greater than its value on the previous iteration. Otherwise, the step size remains the same for the next iteration. Also, after every 1000 iterations the step size is increased by 10 times. The minimization algorithm is stopped either after 15000 iterations or when the step size becomes less then $10^{-14}$. The latter means that the functional can no longer decrease and its minimizer is reached. 


\subsection{Algorithm}

Here, we summarize our algorithm for reconstructing the unknown function $c(x)$ from noisy computationally simulated data $g_{0, \delta}(k)$. Below in this section, we assume that our data have noise and omit the subscript $\delta$ for brevity. Also, all functions in this section are discrete, unless otherwise specified.

1. Estimate the location of target and propagate data if necessary, i.e. if $x_{\text {est }}>0.1$.

2. Calculate the boundary conditions $q_{0}, q_{1}$ in 31 and then $f_{0}, f_{1}$ in 40 as

$$
\begin{gathered}
f_{0, n}=\sum_{m=0}^{N_{k}} \psi_{m, n} q_{0, m}, \quad f_{1, n}=\sum_{m=0}^{N_{k}} \psi_{m, n} q_{1, m}, n=0, \ldots, N-1, \\
f_{0}=\left(f_{0,0}, \ldots, f_{0, N-1}\right)^{T}, f_{1}=\left(f_{1,0}, \ldots, f_{1, N-1}\right)^{T} .
\end{gathered}
$$

3. Define the initial guess $Y_{0}$ for the vector function $Y$ as $Y_{0}=f$ in 42 .

4. Minimize the functional $J_{\lambda, \alpha}(Y)$ in 50 . Then transform the found vector function $Y$ in the vector function $V$ in 115 .

5. Using 29$)$ with $k=\underline{k}$, compute the approximation $\beta_{\text {comp }}$.

6. After averaging $\beta_{\text {comp }}(x)$, determine the coefficient $c_{c o m p}$ as follows:

$$
c_{\text {comp }}=\left\{\begin{array}{cc}
\operatorname{Re}\left(\beta_{\text {comp }}\right)+1.0, & \text { if } \operatorname{Re}\left(\beta_{\text {comp }}\right) \geq \rho \max \left(\operatorname{Re}\left(\beta_{\text {comp }}\right)\right), \\
1.0, & \text { otherwise }
\end{array}\right.
$$

where $\rho \in(0,1)$ is the truncated factor.

Step 6 of the algorithm is a simple post-processing to reconstruct our simulated targets.

\subsection{Reconstruction results for computationally simulated data}

In this section we present the results of reconstructions via the proposed algorithm for the computationally simulated targets with $\widehat{c}_{t r u e}$ and $x_{l o c}$ set as in 108. These results are obtained using the Carleman weight function $e^{-2 \lambda x}$ with the parameter $\lambda=3.0$ in 50 , the regularization parameter $\alpha=0.05$, the discretization parameters $N_{x}=50, N_{k}=3, N=3$, and the truncation factor $\rho=0.5$ in 116). Table 1 lists all reconstructed targets with the maximum value of the computed function,

$$
\widehat{c}_{c o m p}=\max \left(c_{c o m p}\right) .
$$

We define the relative computational error:

$$
\varepsilon_{\text {comp }}=\frac{\left|\widehat{c}_{\text {comp }}-\widehat{c}_{\text {true }}\right|}{\widehat{c}_{\text {true }}} \cdot 100 \% \text {. }
$$

The reconstructed functions $c_{\text {comp }}$ in 116 for some selected targets are shown in Figure 3. We can see that these results are quite accurate ones, given that with the noise level in the data is $5 \%$. 
Table 1: Reconstruction results for simulated targets.

\begin{tabular}{|c|c|c|c||c|c|c|c|}
\hline$\widehat{c}_{\text {true }}$ & $x_{\text {loc }}$ & $\widehat{c}_{\text {comp }}$ & $\varepsilon_{\text {comp }}, \%$ & $\widehat{c}_{\text {true }}$ & $x_{\text {loc }}$ & $\widehat{c}_{\text {comp }}$ & $\varepsilon_{\text {comp }}, \%$ \\
\hline 3.0 & 0.1 & 2.98 & 0.67 & 5.0 & 0.1 & 5.32 & 6.40 \\
& 0.2 & 3.13 & 4.33 & & 0.2 & 5.14 & 2.80 \\
& 0.3 & 2.80 & 6.67 & & 0.3 & 5.11 & 2.20 \\
& 0.4 & 3.17 & 5.67 & & 0.4 & 5.19 & 3.80 \\
\hline 4.0 & 0.1 & 4.28 & 7.00 & 6.0 & 0.1 & 6.19 & 3.17 \\
& 0.2 & 3.95 & 1.25 & & 0.2 & 6.25 & 4.17 \\
& 0.3 & 4.03 & 0.75 & & 0.3 & 6.39 & 6.50 \\
& 0.4 & 4.12 & 3.00 & & 0.4 & 6.47 & 7.83 \\
\hline
\end{tabular}

\subsection{Reconstruction results for experimental data}

Now we demonstrate the reconstruction results for the experimental data. Recall that these data were collected in the field (as opposed to a laboratory) by the Forward Looking Radar of the US Army Research Laboratory [2]. The scheme of data collection is presented on Figure 4. Originally the backscattering time dependent data are measured, one time resolved curve for each target. To obtain the data in the frequency domain, we apply Fourier transform. Since samples of shapes of both time dependent experimental curves and their Fourier transforms can be found in [34, we do not display them here.

The same experimental data were used in our previous works 1, 34, 38, 39, 40. Hence, we do not describe here details of the data collection method as well as the data pre-processing procedure. Instead, we refer to the above mentioned previous works. Especially detailed description can be found in 38, 39. In references [34, 38, 39, 40] these data were treated by the tail functions globally convergent method and in [1] they were treated by the previous version of the convexification method.

Targets of our interest were surrounded by cluttered environment, which is of course a complicating factor for their imaging. Horizontal coordinates of targets were provided by Ground Positioning System (GPS) with a good accuracy. As to the burial depths of targets, they are not of an interest in this specific application. In addition, we had the data for two targets located in air. Hence, the burial depth for these two makes no sense. Furthermore, it is clear from the descriptions of [38, 39] of the data collection process that it is unlikely that an information about burial depths of buried targets can ever be extracted from these data. All what was known to us was that burial depths of targets buried in the ground (dry sand) was a few centimeters. With respect to our algorithm this means that we are not interested in the location of the target. Rather, our interest is in the target/background contrast in the dielectric constant, see (118). Hence, unlike the computationally simulated data, we do not apply here the procedure of section 6.2.

We posses experimental data for five (5) targets. Our a priori information was that two (2) targets, bush and a wood stake, were located in air, and three (3) targets, metallic box, metallic cylinder and plastic cylinder, were buried in a dry sand. We also knew that each experimental data set was collected for a single target only. We introduce the number $c_{b g}$ for the dielectric constant of 


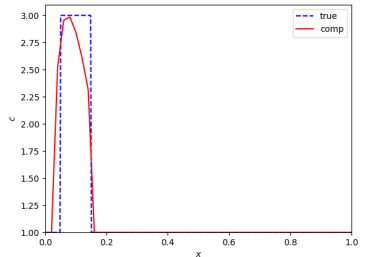

(a)

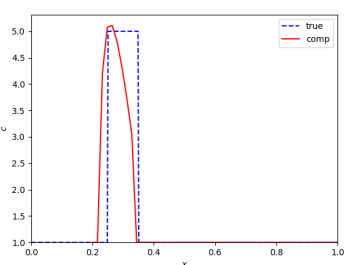

(c)

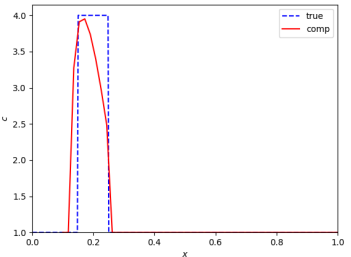

(b)

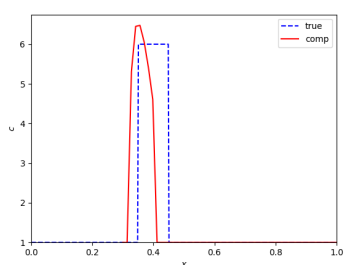

(d)

Figure 3: Reconstruction results for targets with: a) $\widehat{c}_{\text {true }}=3.0, x_{l o c}=0.1, b$ ) $\left.\left.\widehat{c}_{\text {true }}=4.0, x_{l o c}=0.2, c\right) \widehat{c}_{\text {true }}=5.0, x_{l o c}=0.3, d\right) \widehat{c}_{\text {true }}=6.0, x_{l o c}=0.4$

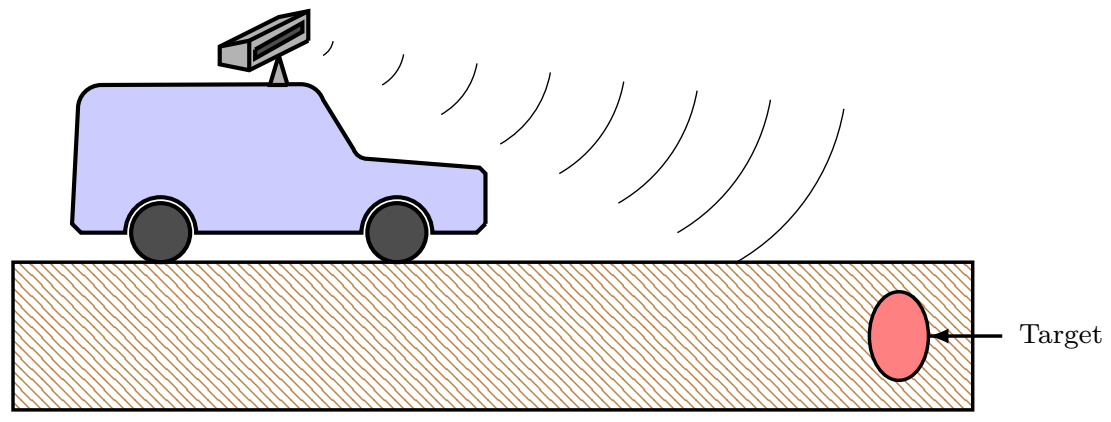

Figure 4: Data collection scheme 
the background, which is $c_{b q}=1.0$ if the target is in air, and $c_{b g} \in[3.0,5.0]$ if the target is buried. Here we use values of the dielectric constant of dry sand, see tables 41 of dielectric constants. Consequently, the computed number $\widehat{c}_{c o m p}=\max c_{c o m p}(x)$ in 117 is an estimation of the contrast between the target and the background $c_{\text {contrast }}$,

$$
c_{\text {contrast }}=\frac{c_{\text {target }}}{c_{b g}} \approx \widehat{c}_{\text {comp }} \text {. }
$$

where $c_{\text {target }}$ is the true dielectric constant of the target and the function $c_{\text {comp }}(x)$ is as in 116 .

Also, we know that the dielectric constant of plastic cylinder is less then the one of sand with $0<c_{\text {contrast }}<1.0$, see "plastic pellets" in [41]. Therefore, we modify 116 for this case as:

$$
c_{\text {contrast }}=\left\{\begin{array}{cc}
\operatorname{Re}\left(\beta_{\text {comp }}\right)+1.0, & \text { if } \operatorname{Re}\left(\beta_{\text {comp }}\right) \leq \rho \min \left(\operatorname{Re}\left(\beta_{\text {comp }}\right)\right), \\
1.0, & \text { otherwise and also if } \operatorname{Re}\left(\beta_{\text {comp }}\right)<-1.0,
\end{array}\right.
$$

where $\rho=0.5$. Here, the condition $\operatorname{Re}\left(\beta_{\text {comp }}\right)<-1.0$ must be applied before the truncation. Next, we introduce the number $\widehat{c}_{e s t}$, which is our estimation of the dielectric constant of the target,

$$
\widehat{c}_{e s t}=c_{b g} \widehat{c}_{c o m p},
$$

where $\widehat{c}_{\text {comp }}=\min \left(c_{\text {comp }}\right)$ for the plastic cylinder, and $\widehat{c}_{\text {comp }}=\max \left(c_{\text {comp }}\right)$ for other targets. A priori differentiation between the plastic cylinder and other targets is performed prior our computations via an analysis of the data, see [39.

The numbers function $\widehat{c}_{\text {comp }}$ as well as estimates of dielectric constant $\widehat{c}_{\text {est }}$ via 119 for our targets are listed in Table 2. Since we did not measure dielectric constants of targets, then the values of $c_{\text {target }}$ were obtained from tables 41 . Also, it was shown in 39 that one can assign large values of dielectric constants to metallic targets as $c_{m e t}:=c_{\text {target }} \in[10,30]$. As to the bush, this target is considered as the hardest one to image since it is obviously a very heterogeneous one due to the presence of many leaves. We took for this case $c_{\text {target }}$ from 42 . Our results are summarized in Table 2

Table 2: Reconstruction results for experimental targets.

\begin{tabular}{|l|c|c|c|c|c|}
\hline Target & Air/Sand & $\widehat{c}_{\text {contrast }}$ & $c_{b g}$ & $\widehat{c}_{\text {est }}$ & $c_{\text {target }}$ \\
\hline Bush & Air & 5.47 & 1.0 & 5.47 & {$[3.0,20.0]$} \\
Wood stake & Air & 3.80 & 1.0 & 3.80 & {$[2.0,6.0]$} \\
Metal box & Sand & 4.91 & {$[3.0,5.0]$} & {$[14.73,24.55]$} & {$[10.0,30.0]$} \\
Metal cylinder & Sand & 4.84 & {$[3.0,5.0]$} & {$[14.52,24.20]$} & {$[10.0,30.0]$} \\
Plastic cylinder & Sand & 0.59 & {$[3.0,5.0]$} & {$[1.77,2.95]$} & {$[1.1,3.2]$} \\
\hline
\end{tabular}

One can see that all values of estimated dielectric constants $\widehat{c}_{e s t}$ in this table are within tabulated limits. Given that dielectric constants of targets were not measured in experiments, Table 2 is a quite encouraging one for the engineering part of this research group (AS and LN). Indeed, results presented in this table indicate that a future software based on the above algorithm might 


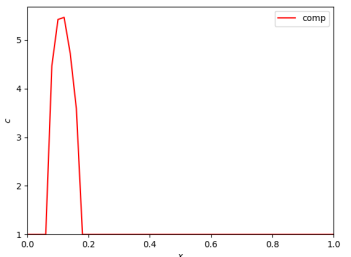

(a)

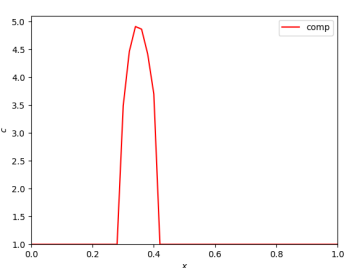

(c)

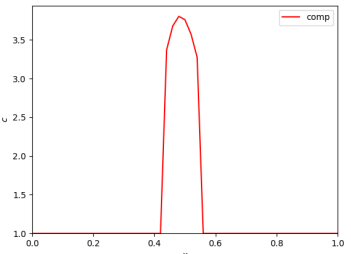

(b)

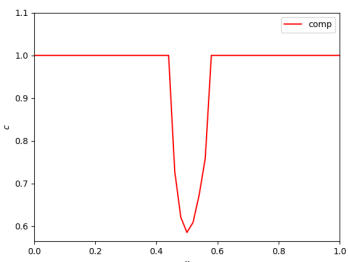

(d)

Figure 5: Reconstruction results for: a) bush, b) wood stake, c) metallic box, d) plastic cylinder

indeed provide rather accurate estimates of dielectric constants of targets of interest. An important additional point of the encouragement of engineers is that these results are obtained for targets surrounded by a realistic cluttered environment. Hence, engineers conjecture that an intriguing opportunity might occur in the future to decrease the false alarm rate, as mentioned in section 1. Certainly more comprehensive studies of large collections of experimental data sets are necessary to verify this conjecture.

\section{Acknowledgements}

The work of M. V. Klibanov and A.E. Kolesov was supported by the US Army Research Laboratory and US Army Research Office grant W911NF-15-1-0233 as well as by the Office of Naval Research grant N00014-15-1-2330.

\section{References}

[1] M. V. Klibanov, A. E. Kolesov, L. Nguyen, and A. Sullivan. Globally strictly convex cost functional for a 1-d inverse medium scattering problem with experimental data. SIAM J. on Applied Mathematics, 77:1733-1755, 2017.

[2] L. Nguyen, D. Wong, M. Ressler, F. Koenig, B. Stanton, G. Smith, J. Sichina, and K. Kappra. Obstacle avoidance and concealed target detection using the Army Research Lab ultra-wideband synchronous impulse reconstruction (UWB SIRE) forward imaging radar. page 65530H, 2007. 
[3] M. Soumekh. Synthetic aperture radar signal processing with MATLAB algorithms. John Wiley \& Sons, New York, 1999.

[4] L. Beilina and M. V. Klibanov. Globally strongly convex cost functional for a coefficient inverse problem. Nonlinear Analysis: Real World Applications, $22: 272-288,2015$.

[5] M. V. Klibanov and O. V. Ioussoupova. Uniform strict convexity of a cost functional for threedimensional inverse scattering problem. SIAM J. Math. Anal., 26:147-179., 1995.

[6] M. V. Klibanov. Global convexity in a three-dimensional inverse acoustic problem. SIAM Journal on Mathematical Analysis, 28:1371-1388, 1997.

[7] M. V. Klibanov and A. Timonov. Carleman Estimates for Coefficient Inverse Problems and Numerical Applications. de Gruyter, Utrecht, 2004.

[8] M. V. Klibanov and N. T. Thành. Recovering dielectric constants of explosives via a globally strictly convex cost functional. SIAM Journal on Applied Mathematics, 75:518-537, 2015.

[9] M. V. Klibanov and A. E. Kolesov. Convexification of a 3-d coefficient inverse scattering problem. Computers and Mathematics with Applications, published online, 2018.

[10] A. B. Bakushinskii, M. V. Klibanov, and N. A. Koshev. Carleman weight functions for a globally convergent numerical method for ill-posed cauchy problems for some quasilinear pdes. Nonlinear Analysis: Real World Applications, 34:201-224, 2017.

[11] M. V. Klibano. Carleman weight functions for solving ill-posed cauchy problems for quasilinear pdes. Inverse Problems, 31:125007, 2015.

[12] M. V. Klibanov, J. Li, and A.G. Yagola. Numerical solution of an illposed cauchy problem for a quasilinear parabolic equation using a carleman weight function. J. Inverse and Ill-Posed Problems, 24:761-776, 2016.

[13] J.A. Scales, M.L. Smith, and T.L. Fischer. Global optimization methods for multimodal inverse problems. Journal of Computational Physics, 103(2):258-268, 1992.

[14] A. Bukhgeim and M. Klibanov. Uniqueness in the large of a class of multidimensional inverse problems. Soviet Math. Doklady, 17:244-247, 1981.

[15] M. V. Klibanov. Carleman estimates for global uniqueness, stability and numerical methods for coefficient inverse problems. Journal of Inverse and Ill-Posed Problems, 21:477-560, 2013.

[16] L. Beilina and M. V. Klibanov. Approximate Global Convergence and Adaptivity for Coefficient Inverse Problems. Springer, 2012.

[17] M. Bellassoued and M. Yamamoto. Carleman estimates and applications to inverse problems for hyperbolic systems. Springer, Japan, 2017. 
[18] M.V. Klibanov, D.-L. Nguyen, L.H. Nguyen, and H. Liu. A globally convergent numerical method for a $3 \mathrm{~d}$ coefficient inverse problem with a single measurement of multi-frequency data. Inverse Problems and Imaging, 12:493-523, 2018.

[19] M. de Buhan L. Baudouin and S. Ervedoza. Convergent algorithm based on carleman estimates for the recovery of a potential in the wave equation. SIAM J. on Numerical Analysis, 55:1578-1613, 2017.

[20] M. V. Klibanov. Convexification of restricted dirichlet-to-neumann map. J. Inverse and Ill-Posed Problems, 25:669-685, 2017.

[21] M. V. Klibanov, J. Li, and W. Zhang. Electrical impedance tomography with restricted dirichlet-to-neumann map data. 2018.

[22] H. Ammari, J. Garnier, W.Jing, M. Lim H.Kang, K. Sølna, and H. Wang. Mathematical and statistical methods for multistatic imaging. Springer, New York, 2013.

[23] H. Ammari, Y.T. Chow, and J. Zou. The concept of heterogeneous scattering and its applications in inverse medium scattering. SIAM J. Math. Anal., 46:2905-2935, 2014.

[24] H. Ammari, Y.T. Chow, and J. Zou. Phased and phaseless domain reconstruction in inverse scattering problem via scattering coefficients. SIAM J. Appl. Math., 76:1000-1030, 2016.

[25] G. Chavent. Nonlinear Least Squares for Inverse Problems - Theoretical Foundations and Step-by-Step Guide for Applications. Springer, 2009.

[26] A. Goncharsky and S. Romanov. Supercomputer technologies in inverse problems of ultrasound tomography. Inverse Problems, 29:075004, 2013.

[27] A. V. Goncharsky and S. Y. Romanov. Iterative methods for solving coefficient inverse problems of wave tomography in models with attenuation. Inverse Problems, 33:025003, 2017.

[28] K. Ito, B. Jin, and J. Zou. A direct sampling method to an inverse medium scattering problem. Inverse Problems, 28:025003, 2012.

[29] K. Ito, B. Jin, and J. Zou. A direct sampling method for inverse electromagnetic medium scattering. Inverse Problems, 29:095018, 2013.

[30] J. Li, H. Liu, and Y. Wang. Recovering an electromagnetic obstacle by a few phaseless backscattering measurements. Inverse Problems, 33:035011, 2017.

[31] S. I. Kabanikhin, A. D. Satybaev, and M. Shishlenin. Direct Methods of Solving Multidimensional Inverse Hyperbolic Problem. VSP, Utrecht, 2004.

[32] S. I. Kabanikhin, N. S. Novikov, I. V. Osedelets, and M. Shishlenin. Fast toeplitz linear system inversion for solving two-dimensional acoustic inverse problem. J. Inverse Ill-Posed Problems, 23:687-700, 2015. 
[33] S. I. Kabanikhin, K. K. Sabelfeld, N. S. Novikov, and M. A. Shishlenin. Numerical solution of coefficient recovering for a wave equation by a stochastic projection methods. J. Inverse Ill-Posed Probl., 23:189-203, 2015.

[34] L. Nguyen M.V. Klibanov, L.H. Nguyen and A. Sullivan. A globally convergent numerical method for a 1-d inverse medium problem with experimental data. Inverse Problems and Imaging, 10:1057-1085, 2016.

[35] D. Colton and R. Kress. Inverse Acoustic and Electromagnetic Scattering Theory. Springer, New York, 2013.

[36] A. B. Bakushinskii, M.Yu. Kokurin, and M.M. Kokurin. Regularization Algorithms for Ill-Posed Problems. De Guyter, Berlin, 2018.

[37] M. V. Klibanov A. V. Kuzhuget. Global convergence for a 1-d inverse problem with application to imaging of land mines. Applicable Analysis, 89:125-157, 2010.

[38] A.L. Karchevskii, M.V. Klibanov, L. Nguyen, N. Pantong, and A. Sullivan. The krein method and the globally convergent method for experimental data. Applied Numerical Mathematics, 74:111-127, 2013.

[39] A.V. Kuzhuget, L. Beilina, M.V. Klibanov, A. Sullivan, L. Nguyen, and M.A. Fiddy. Blind backscattering experimental data collected in the field and an approximately globally convergent inverse algorithm. Inverse Problems, 28(9):095007, 2012.

[40] A.V. Kuzhuget, L. Beilina, M.V. Klibanov, A. Sullivan, L. Nguyen, and M.A. Fiddy. Quantitative Image Recovery From Measured Blind Backscattered Data Using a Globally Convergent Inverse Method. IEEE Transactions on Geoscience and Remote Sensing, 51(5):2937-2948, may 2013.

[41] http://www.clippercontrols.com/pages/ Dielectric-Constant-Values.html.

[42] H. T. Chuah, K. Y. Lee, and T. W. Lau. Dielectric constants of rubber and oil palm leaf samples at x-band. IEEE Transactions on Geoscience and Remote Sensing, 33:221-223, 1995. 